\title{
A Robust Bank Asset Allocation Model Integrating Credit-Rating Migration Risk and Capital Adequacy Ratio Regulations
}

\author{
Dawen Yan1, Xiaohui Zhang', Mingzheng Wang ${ }^{3 *}$
}

1. School of Mathematical Science and Faculty of Management \& Economics, Dalian University of Technology, Dalian 116024, China. Email: dawenyan@ dlut.edu.cn.

2. Business School, University of Exeter, Exeter, EX4 4PU, United Kingdom. Email: x.zhang1@exeter.ac.uk

3. School of Management, Zhejiang University, Hangzhou 310058, China. Email: wangmzh@zju.edu.cn.

* Corresponding author

\begin{abstract}
In this paper, we consider a bank asset allocation problem with uncertain migration risk of credit ratings and capital adequacy ratio (CAR) regulations. In the practical scenarios, the future market values of each risky asset are largely affected by outer complex environments. We only observe the information about their first-moment and marginal second-moment of year-ahead market value of each loan asset. Based on these scenarios, we propose a new distributionally robust optimization model with the chance constraint characterized by uncertain capital adequacy ratio. Following the duality theory in infinite-dimensional optimization problem and the theory of conic linear optimization model, we can reformulate the original problem into a tractable linear deterministic semi-definite programming (SDP) model. By using this tractable linear SDP model, we can provide a robust asset allocation policy to bank managers. Further, we conduct a simulation study to illustrate the application of our method under two different economic conditions, a downward condition and an upward condition. Then a series of sensitivity tests is applied to examine the impacts of various factors, including safety probability, target CAR and recovery rate, on the optimal asset allocations. We also compare the performance of our model and the CVaR model. We demonstrate our model provides an efficient way to deal with the trade-off between expected return and CAR.
\end{abstract}

Keywords: Asset allocation, Credit rating migration, Capital adequacy ratio, Distributionally robust optimization, Chance constraint

JEL classification: G21; C61

\section{Introduction}

Asset allocation plays an important role in bank management. Banks are encouraged to invest diversely, especially to invest in assets less likely to default on. An efficient asset allocation strategy can make profits and avoid risks for a bank. One of the core activities in asset allocation conducted by banks is credit risk management. Credit risk is the potential that a bank borrower or counterparty will fail to meet its obligations in accordance with agreed terms. Credit risk can be caused either by counterparty default, or by the shrink of market value due to credit quality migration of the counterparty (Andersson et al. 2001; Altman and Saunders 1997; Iscoe et al. 2012).

In practice, bank managers tend to invest in borrowers with relatively higher credit rating at the beginning of investment period to reduce the credit risk. However, the credit ratings of firm loans and other risky assets may migrate within the maturity, because of the changes in firm-level determinants, trend of industry development, and macroeconomic conditions (Tang and Yan 2010; Figlewski et al. 2012). For example, according to the data released by China Banking Regulatory Commission, the balance of non-performing loans of Chinese commercial banks was approximately 400 billion in the third quarter of 2011, and it had been increased to 1.34 trillion by the end of the fourth quarter of 2017. In fact, the majority of the non-performing loans has ever transformed from performing loans, due to credit risk migration from low risk to high risk within 
the maturity. The uncertainty of credit rating migration is a challenge that bank managers need to overcome in order to make correct investment decisions and to avoid misallocation and losses.

It is well known that, in the last three decades, the Basel Committee on Banking Supervision (BCBS) has consistently increased the minimal capital requirement for banks to avoid excess leverage and to assure capital adequacy and financial stability. Basel I Accord (1988) requires banks with an international presence to maintain a minimum of 8\% Capital Adequacy Ratio (CAR). Basel II Accord (2004) revises the international standards for measuring the adequacy of a bank's capital through risk assessment. Banks maintain an adequate capital ratio to signal soundness to the market and to satisfy the expectations of rating agencies (Shim 2013). Furthermore, it has been found that well capitalized banks weathered the 2007-08 financial crisis better (see for example, Huang and Xiong 2015; Guidara et al. 2013). After the 2007-08 financial turmoil, Basel III Accord (2010) put forward a more stringent regulatory standard. In addition to the minimum $8 \%$ of CAR, banks are mandated to hold a capital conservation buffer of $2.5 \%$ above the regulatory capital requirement. In this context, an optimal bank asset portfolio allocation needs to comply with the CAR regulatory requirement. Good examples can be found in Mukuddem-Petersen and Petersen (2008), and Muller and Witbooi (2014).

In fact, credit rating migration, minimum capital charge and capital adequacy ratio regulation are correlated with each other (See Altman and Saunders 2001; Peura and Jokivuolle 2004; Allen et al. 2016). Credit rating migration causes the assets' market value change, and ultimately causes the changes in the bank's target capital requirements and capital adequacy ratio (Altman and Saunders 2001; Altman et al. 2002; Peura and Jokivuolle 2004). However, to the best of the authors' knowledge, no previous study, which solves the bank asset allocation optimization problem, not only takes into account of the credit rating migration risk but also subjects the asset allocation to the capital adequacy ratio requirement.

In this study we propose a bank asset allocation model. The optimal asset allocation suggested by our model maximizes the expected portfolio rate of return, under the context that there are uncertain credit rating migrations and the regulatory requirement on capital adequacy ratio (CAR) needs to be met. In our model, we use a distributionally robust chance constrained optimization approach. Comparing to the conventional asset allocation model, our method only requires partial information, but not the entire, of the credit loss/return distribution. Therefore our method is more robust to the estimated credit loss/return distribution (Charnes and Cooper 1959; Miller and Wagner 1965; Nemirovski and Shapiro 2006). Furthermore, it can be applied to firms or industries, for which no accurate entire distribution can be estimated due to lack of historical data. We conduct a simulation study to illustrate the application of our method under two different economic conditions, an upturn and a downturn. A series of sensitivity tests is applied to examine the impacts of various factors, including safety probability, target CAR and recovery rate, on the optimal asset allocations. Results from the simulation study show the efficiency of our model in terms of the trade-off between sacrificing investment return and meeting the regulatory requirement of CAR. We also compare the performance of our method with that of the Conditional Value-at-Risk ( $\mathrm{CVaR})$ optimization model, in terms of meeting the regulatory requirement of CAR.

The rest of this paper is organized as follows. In Section 2 we briefly review the modern bank asset allocation theory, discuss the Credit-Metrics method and how to apply it to compute the first-order and marginal second-order moment of one-year-ahead market value of a loan. Section 3 constructs a distributionally robust asset allocation model, which takes into account of the random credit rating migration risks and has a constraint to assure the target CAR. This model is further converted to a tractable linear semidefinite programming (SDP) problem through using the duality theory in infinite-dimensional optimization problem and the theory of conic linear moment problems. In Section 4, we conduct a simulation study to illustrate our method. A range of sensitivity tests are conducted to examine the impact of various factors on the optimal asset allocation. We also compare the performance of our model with that of a widely used asset 
allocation method, i.e. the $\mathrm{CVaR}$ model, in terms of expected portfolio return and assurance of CAR. Section 5 concludes.

\section{Background}

\subsection{Literature review on modern bank asset allocation}

Modern bank asset allocation theory has established and developed based on the classical mean-variance formulation pioneered by Markowitz (1952) and rooted in the stochastic optimization theory and the bank risk management theory (see Kane and Malkiel 1965; Kusy and Ziemba 1986; Oguzsoy and Gu 1997; Kaplanski and Levy 2015). Different risk measures, such as Value at Risk (VaR), Conditional Value at Risk (CVaR) and Lower partial moment (LPM) etc., have been widely applied to the asset allocation of banks. The VaR risk measure developed originally by the firms for their risk-management purposes attempts to measure the loss on a portfolio over a specified period that will only be exceeded on a given fraction of occasions (typically $1 \%$ or $5 \%$ ). Mathematically, VaR has serious limitations. For example, in the case of a finite number of scenarios, it is a non-smooth and non-convex function, causing the difficulty in controlling and optimizing. The undesirable property of lack of sub-additivity in VaR makes it inapplicable in practice. CVaR, which is known also as Mean Excess Loss, Mean Shortfall, or Tail VaR, is introduced by Artzner et al. (1999). CVaR is a coherent risk measure and defined as the expected loss conditional on losses exceeding VaR. It is considered to be a more consistent risk measure than VaR. CVaR greatly facilitates the application of optimization to risk management, i.e. optimizing with an objective based on $\mathrm{CVaR}$, and the calculations often come down to linear programming (Saunders et al. 2007). In a VaR or CVaR model, the risk is estimated based on the assumption that the uncertain parameter's distribution is known and independent over time, which can be estimated or simulated based on historical data (Jackson et al. 1998; Allen et al. 2016). However, in practice, we do not always have sufficient data to accurately estimate the entire distribution. The distribution uncertainty may cause false estimation on risk measure and lead to wrong decisions (Delage and Ye 2010; Kolm et al. 2014).

Distributionally robust chance constrained optimization method has been increasingly applied in portfolio selection, as it only requires partial information of the uncertain parameter's distribution (see El Ghaoui et al 2003; Zymler et al. 2013A; Sun et al. 2017). The conventional distributionally robust chance constrained models are formulated and solved based on a set of probability distributions consistent with the known properties of the uncertain parameter's distribution, such as the first and the second moment (El Ghaoui et al 2003; Calafiore and EI Ghaoui 2006; Zymler et al. 2013A, 2013B; Sun et al. 2017). The covariance of the uncertain parameter impact on the degree of dispersion of the uncertain parameter and are important for the subsequent risk measure estimation and asset allocation. However, accurately estimating them is difficult. Take our study as an example, the uncertain parameter is a future mark-to-market value vector of the risky assets, which is highly correlated with the credit rating migration. But it is hard to accurately estimate its covariance, because neither the joint credit migration nor the joint market value is directly observable process. The link needs to be inferred to observable correlations, such as equity returns (see Bucay and Rosen 1999; Das et al. 2007; Pu and Zhao 2012, Iscoe et al. 2012; Hilscher and Wilson 2017). But this approach may lead to inaccurate estimations of the correlations and misallocation of asset portfolio (Delage and Ye 2010, Dorfleitner and Pfister 2013).

Another very important recent research line in the area of bank asset allocation is the large-scale portfolio selection problem. For example, Sirignano et al. (2016) consider a large-scale portfolio selection and propose an approximate optimization approach that reduces the computational cost significantly. Sirignano and Giesecke (2018) discuss the default and prepayment risk management problem for a large pool of risky loans.

Due to the importance of credit rating migration in credit risk management of financial institutions, a considerable amount of effort has been devoted to asset portfolio optimization under credit migration. In 
general, three steps are involved in the optimizations, i.e. (1) estimate a credit loss (or return) distribution, which depends on the underlying credit loss model; (2) choose and estimate a credit risk measure; and (3) apply an asset allocation optimization model. For example, Bucay and Rosen (1999) use the Credit-Metrics methodology (Gupton et al. 1997) and a Monte Carlo simulation of joint obligor credit states to obtain the distribution of portfolio loss. Based on a credit risk distribution generated by Monte Carlo simulations (Bucay and Rosen 1999), Andersson et al. (2001) minimize CVaR risk measure of portfolio credit loss, subject to trading and return constraints, through linear programming. Taking into account of credit migration, Iscoe et al. (2012) estimate the portfolio loss distribution using the Monte Carlo simulation approach. They then evaluate several alternative formulations for minimizing portfolio credit risk, in terms of VaR and CVaR. Singh and Dharmaraja (2017) consider a bond portfolio optimization problem based on the risk measure of $l_{\infty}$-norm, assuming that the assets' credit rating migrations are driven by discrete time Markov chain model and are independent to each other. Gao et al. (2017) investigate the dynamic mean lower partial moment (LPM) and mean CVaR portfolio optimization problems in continuous-time.

\subsection{Credit-Metrics method}

The credit rating of a risky asset may randomly migrate before the maturity date, which influences not only the asset's market value but also the capital adequacy ratio of the bank holding the asset. Three possible scenarios of credit rating transition of a risky asset are presented in Figure 1, where AAA, AA, A, BBB, BB, B and $\mathrm{CCC}$ denote 7 non-default rating categories and $\mathrm{D}$ denotes the default rating.

Credit-Metrics approach has been widely used in both theory and practice of credit risk management to assess single-asset risk or portfolio risk due to defaults and changes in borrowers' credit rating (Gupton et al. 1997; Singh and Dharmaraja 2017). In this paper we apply Credit-Metrics approach to compute the first-order and marginal second-order moment of one-year-ahead market value of a risky asset allowing for the aforementioned random credit rating migration. In particular, the risky assets' one-year-ahead market values are the uncertainty parameter in the distributionally robust chance constrained optimization model used in this study.

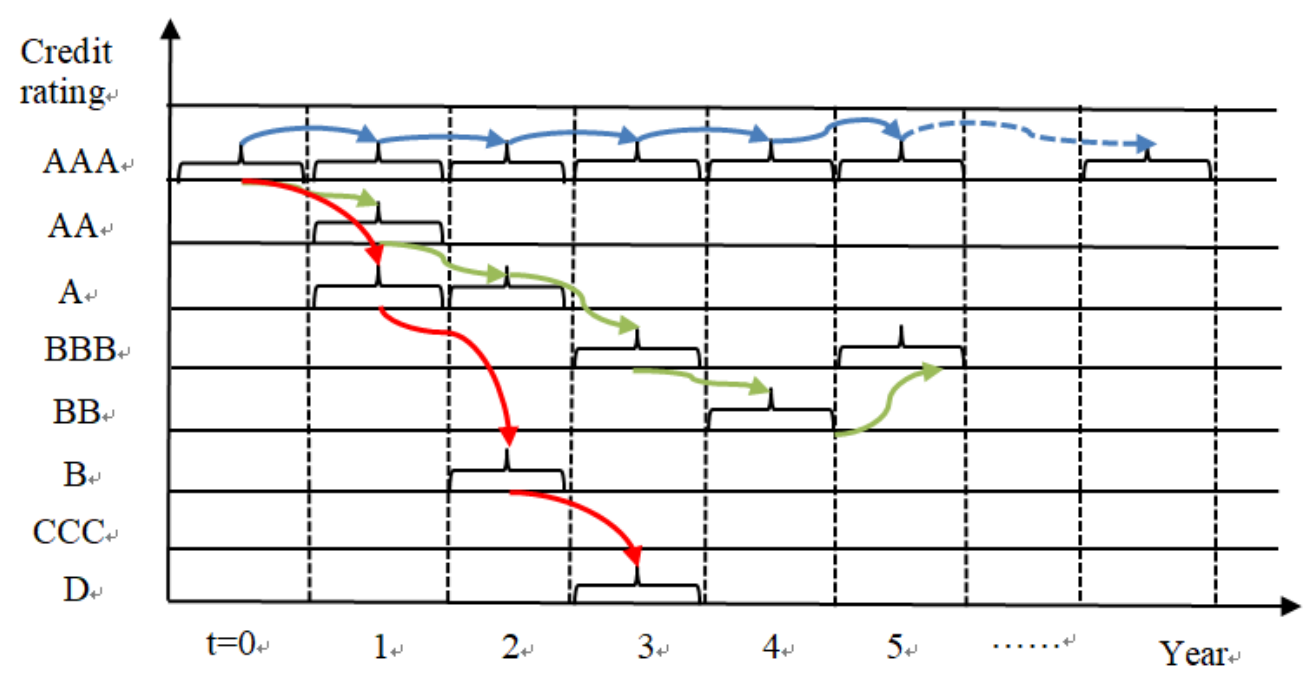

Fig. 1 Credit rating migration path

One key step of the Credit-Metrics method is to compute the multi-year migration probability of a possible credit rating migration path before the maturity date for an individual obligator. The one-year credit migration probabilities of an asset from one rate to another can be obtained from the Standard \& Poor's transition matrix. For example, Table 1 and Table 2 show the one-year corporate transition rates for the US in 2007 and 2013, respectively. If we suppose the credit rating migrations follow a homogeneous Markov chain, the multi-year migration probabilities are the product of the one-year transition rates. This assumption makes it tractable to 
generate forecasts of the expected future market values and variances of risky assets, which are required for our model as its input parameters. We acknowledge it is a strong assumption, though it is widely adopted because of convenience. Previous studies have shown the presence of non-Markovian behaviour of a rating process (see Altman 1998; Duffie et al. 2007). In fact, the assumption of homogenous Markov process can be relaxed at the cost of estimation parsimony (Frydman and Schuermann 2008; Pfeuffer et al. 2018). Given the objective of this paper is to propose a new robust asset allocation model, estimating the credit rating migration probabilities in different setups (such as a non-Markov setup) is out of the scope, but a promising extension, of the current study. For simplicity, we follow the common practice to assume the credit rating migrations follow a homogeneous Markov chain.

Another key step of the Credit-Metrics method is to compute the one-year-ahead market value of an asset. To do so, we use the forward rates implied by current term structure in each of the seven non-default states. For example, the forward rates of year 2007 in the US are shown in Table 3. JP Morgan also provides forward rates to the public regularly. In the case of default, the market value of a risky asset (for example, a corporate loan) depends on its recovery rate. Recovery rates on defaulted loans play a critical role in credit risk modeling and are mainly determined by three aspects, i.e. loan characteristics, macroeconomic conditions and industry characteristics (Araten et al. 2004; Khieu et al. 2012).

\subsection{Notations and basic assumptions}

Before proceeding to our modeling method, it is convenient to introduce some assumptions and notations that will be used throughout this paper at this point. First of all, the unit of time in this study is year. We consider a portfolio of various corporate loans and risk-free assets. Suppose that a bank is going to allocate its fund in $l$ risk-free assets and $n$ fixed-rate firm loans each year. In particular, some assumptions are listed below.

Assumption 1: all loans will be held to maturity and not be sold, if no default occurs.

Assumption 2: each single loan's credit rating process before maturity follows a time-homogenous finite state space Markov chain.

Assumption 3: one-year-ahead market value of bank total liabilities is assumed to be constant and equal to the book value of total liabilities in the bank balance sheet at the time of making investment decision. In fact, it is complicated to calculate the future market value of different types of bank liabilities, such as customer deposits, interbank borrowing and market debts (Hugonnier and Morellec 2017). Our main objective of this paper is to address assets allocation problem in the context with uncertain credit rating migration. Therefore to avoid unnecessary complexity, in this study, we assume constant liabilities.

Some notations are stated as follows.

$S$ : state space of credit ratings for risky assets. In this case study, we consider eight credit rating categories (including default) i.e. $S=\{\mathrm{AAA}, \mathrm{AA}, \mathrm{A}, \mathrm{BBB}, \mathrm{BB}, \mathrm{B}, \mathrm{CCC}, \mathrm{D}\}$, where $\mathrm{D}$ denotes default.

$n_{k} \in \mathrm{Z}_{+}$: the maturity of the $k$-th risky asset, where $\mathrm{Z}_{+}$denotes the set of positive integers. $n_{k}(k=1,2, \ldots, n)$ are predetermined values.

$R_{k}$ : the expected rate of return for risky asset $k$ in the absence of credit migration. If the risky asset is loan, $R_{k}$ is its annual interest rate.

$X_{i}^{k}$ : credit rating of the $k$-th risky asset at the $i$-year-end, which is a random variable and $i=0,1, \ldots, n_{k}$.

$C_{0}{ }^{k}$ : the initial credit rating of the $k$-th risky asset, where $C_{0}{ }^{k} \in S \backslash\{\mathrm{D}\}$ is a predetermined grade.

$C_{i}{ }^{k} \in S$ : a possible rating state of the $k$-th loan at the $i$-year-end. That is to say, $C_{i}{ }^{k}$ is a realization value of the random variable of $X_{i}^{k}$, where $i=0,1, \ldots, n_{k}$.

$R R_{k}$ : the recovery rate of the $k$-th loan under the default situation, which is related to the term and the collateral information of the debt.

$T_{k} \geq 1$ : the default period of the $k$-th risky asset and random variable. 
$F_{k j}\left(T_{k}\right)$ : cash flow from the $k$-th risky asset at the $j$-year-end, where $j=1,2, \ldots, \min \left\{T_{k}, n_{k}\right\}$. In this paper, we assume that the amount repaid at each year-end before the maturity is interest and the last repayment is sum of principle and interest for each loan if no default occurs. We also assume that the bank takes back a fraction of $k$-th loan principle at time of last payment according to the recovery rate of $R R_{k}$ if default occurs at time $T_{k}$. If the principle of each loan is assumed to be one unit of currency, $F_{k j}\left(T_{k}\right)$ can be described as

$$
F_{k j}\left(T_{k}\right)=\left\{\begin{array}{lll}
R_{k}, & j=1,2, \ldots, & \min \left\{T_{k}, \mathrm{n}_{k}\right\}-1 \\
1+R_{k}, & j=n_{k}, \text { if } \quad T_{k}>n_{k} \\
R R_{k}, & j=T_{k}, \text { if } \quad T_{k} \leq n_{k}
\end{array} .\right.
$$

In (1), the conditions of $T_{k}>n_{k}$ and $T_{k} \leq n_{k}$ correspond to the non-default case and default case respectively.

$f_{C_{i}^{k}}^{1, i+1}: i$-year forward rate from 1-year-end to $(i+1)$-year-end for the $k$-th risky asset whose credit rating is $C_{i}^{k}$ at the 1-year-end and remains unchanged until $(i+1)$-year-end. Its value can be obtained from the term structure of forward rate at every non-default grade listed in the Table 3.

$f_{C_{i}^{k}}^{i, i+1}: 1$-year forward rate from $i$-year-end to $(i+1)$-year-end for the $k$-th risky asset whose credit rating is $C_{i}^{k}$ at the $i$-year-end.

When the credit rating of $k$-th risky asset remains unchanged, investing one unit of currency in the asset for $i$ years beyond 1 -year-end at the forward rate $f_{C_{i}^{k}}^{1, i+1}$ must result in the same final payment as investing for $i$-1 years beyond 1 -year-end at $f_{C_{i}^{k}}^{1, i}$, and for 1 year beyond $i$-year-end at $f_{C_{i}^{k}}^{i, i+1}$ (non-arbitrage principle). Mathematically,

$$
\left(1+f_{C_{i}^{k}}^{1, i+1}\right)^{i}=\left(1+f_{C_{i}^{k}}^{1, i}\right)^{i-1}\left(1+f_{C_{i}^{k}}^{i, i+1}\right) .
$$

Thus, 1-year forward rate from $i$-year-end to $(i+1)$-year-end for $k$-the risky asset $f_{C_{i}^{*}}^{i, i+1}$ can be calculated by using equation (2) and the corresponding forward rate values in $C_{i}{ }^{k}$ row of Table 3.

$d_{k j}\left(\left\{C_{i}^{k}\right\}_{i=1}^{j-1}\right):(j-1)$-year discount factor from the $j$-year-end $(j \geq 1)$ to the 1 -year-end, which is determined by the credit rating migration path over $j-1$ years of $k$-th loan and implied forward rates. Then the discount factors satisfy the following equations:

$$
d_{k j}\left(\left\{C_{i}^{k}\right\}_{i=1}^{j-1}\right)=\left\{\begin{array}{ll}
1, & j=1 \\
\prod_{i=1}^{j-1}\left(1+f_{C_{i}^{k}}^{i, i+1}\right)^{-1}, & j=2, \ldots, \min \left\{T_{k}, n_{k}\right\}
\end{array} .\right.
$$

Taking (2) into (3), then discount factors can be written as follows:

$$
\begin{aligned}
& d_{k j}\left(\left\{C_{i}^{k}\right\}_{i=1}^{j-1}\right)= \begin{cases}1, & j=1 \\
\prod_{i=1}^{j-1} \frac{\left(1+f_{c_{i}^{1}}^{1, i}\right)^{i-1}}{\left(1+f_{c_{i}^{k}}^{1, i+1}\right)^{i}}, & j=2, \ldots, \min \left\{T_{k}, n_{k}\right\} .\end{cases} \\
& \xi_{k}\left(\left\{X_{j}^{k}\right\}_{j=1}^{\min \left\{T_{k}, n_{k}\right\}}\right)=\sum_{j=1}^{\min \left\{T_{k}, n_{k}\right\}} F_{k j}\left(T_{k}\right) d_{k j}\left(\left\{X_{i}^{k}\right\}_{i=1}^{j-1}\right): \text { one-year-ahead market value of one unit of }
\end{aligned}
$$
currency invested in the $k$-th loan, which is discounted value of $\min \left\{T_{k}, n_{k}\right\}$ year earnings stream $F_{k j}\left(T_{k}\right)$, 
corresponding discount factor $d_{k j}\left(\left\{X_{i}^{k}\right\}_{i=1}^{j-1}\right)$. It is denoted also as $\xi_{k}$ for brevity. Obviously, the value of $\xi_{k}$ depends on the credit rating migration situations before the end of the contract, either reaching to the maturity or defaulting. Once the credit rating of the $k$-th loan at the $i$-year-end $X_{i}^{k}$ is realized as $C_{i}^{k}$, i.e. $X_{i}^{k}=C_{i}^{k}$, then $d_{k j}\left(\left\{X_{i}^{k}\right\}_{i=1}^{j-1}\right)$ can be determined by formula (4).

\subsection{The expected future market value and variance for each risky asset}

According to the definition of $T_{k}$, there are two possible scenarios:

1) $\left\{T_{k}>n_{k}\right\}=\left\{X_{i}^{k} \in S \backslash\{D\}, i=0,1, \ldots, n_{k}\right\}=\{$ default never occurred for the $k$-th risky asset in maturity\};

2) $\left\{T_{k}=t_{k}, 1 \leq t_{k} \leq n_{k}\right\}=\left\{X_{i}^{k} \in S \backslash\{D\}, i=0,1, \ldots, t_{k}-1, X_{t_{k}}^{k}=D\right\}=\left\{\right.$ default occurred at the end of the $t_{k}$-th year for the $k$-th risky asset\}.

Let $\mu_{k}$ denote the expected year-ahead market value of unit capital invested in the $k$-th loan. It is

$$
\begin{aligned}
\mu_{k} & =E\left(\xi_{k}\left(\left\{X_{j}^{k}\right\}_{j=1}^{\min \left\{T_{k}, n_{k}\right\}}\right)\right) \\
& =E\left(\xi_{k} \mid T_{k}>n_{k}\right) P\left(T_{k}>n_{k}\right)+\sum_{t_{k}=1}^{n_{k}} E\left(\xi_{k} \mid T_{k}=t_{k}\right) P\left(T_{k}=t_{k}\right)
\end{aligned}
$$

The first part of $\mu_{k}$, as shown in (5), is corresponding to the "non-default migration case" and is

$$
E\left(\xi_{k} \mid T_{k}>n_{k}\right) P\left(T_{k}>n_{k}\right)=\sum_{\left\{C_{j}^{k}\right\}_{j=1}^{n_{k} \in S \backslash\{D\}}} \xi_{k}\left(\left\{C_{j}^{k}\right\}_{j=1}^{n_{k}}\right) P\left(\left\{X_{j}^{k}=C_{j}^{k}\right\}_{j=1}^{n_{k}}\right) .
$$

For $T_{k}>n_{k}$,

$$
\xi_{k}\left(\left\{C_{j}^{k}\right\}_{j=1}^{n_{k}}\right)=\sum_{j=1}^{n_{k}} F_{k j}\left(T_{k}\right) d_{k j}\left(\left\{C_{i}^{k}\right\}_{i=1}^{j-1}\right)=\sum_{j=1}^{n_{k}-1} R_{k} d_{k j}\left(\left\{C_{i}^{k}\right\}_{i=1}^{j-1}\right)+\left(1+R_{k}\right) d_{k n_{k}}\left(\left\{C_{i}^{k}\right\}_{i=1}^{n_{k}-1}\right)
$$

Consider the green credit rating path in Figure 1 as an example to show how to compute year-ahead market value under a possible non-default migration path. Suppose the 5-year loan has a $8 \%$ annual interest rate, its non-default rating transition path is $A A A(j=0) \rightarrow A A(j=1) \rightarrow A(j=2) \rightarrow B B B(j=3) \rightarrow B B(j=4) \rightarrow B B B(j=5)$, then corresponding discount factors are calculated as $d_{k 1}=1 ; d_{k 2}=0.9648 ; d_{k 3}=0.9195 ; d_{k 4}=0.8641 ; d_{k 5}=0.7945$. Therefore, year-ahead market value of unit capital invested to the loan under this designated migration path is $\xi_{k}=1.1580$ by (7).

Under Assumption 2, the probability of non-default migration path in (6) with credit ratings of $C_{j}^{k}$ at $j$ year-end, $j=1,2, \ldots, n_{k}$, is

$$
P\left(\left\{X_{j}^{k}=C_{j}^{k}\right\}_{j=1}^{n_{k}}\right)=\prod_{j=1}^{n_{k}-1} P\left(X_{j+1}^{k}=C_{j+1}^{k} \mid X_{j}^{k}=C_{j}^{k}\right) P\left(X_{1}^{k}=C_{1}^{k}\right) .
$$

All the one-year conditional transition probabilities can be founded in Table 1 . The value of $P\left(X_{1}{ }^{k}=C_{1}{ }^{k}\right)$ equals the conditional probability of $P\left(X_{1}{ }^{k}=C_{1}{ }^{k} \mid X_{0}{ }^{k}=C_{0}{ }^{k}\right)$ which represents the possibility from original credit rating of $C_{0}{ }^{k}$ to rating of $C_{1}{ }^{k}$ at 1-year-end. It is also attainable from Table 1.

As for the second part of (5), because default happened, it can be computed as 


$$
\sum_{t_{k}=1}^{n_{k}} E\left(\xi_{k} \mid T_{k}=t_{k}\right) P\left(T_{k}=t_{k}\right)=\sum_{t_{k}=1}^{n_{k}} \sum_{C_{1}^{k}, \ldots, C_{t_{k}-1}^{k} \in S \backslash\{D\}} \xi_{k}\left(\left\{C_{j}^{k}\right\}_{j=1}^{t_{k-1}} \wedge D\right) P\left(\left\{X_{j}^{k}=C_{j}^{k}\right\}_{j=1}^{t_{k}-1} \cap\left\{X_{t_{k}}^{k}=D\right\}\right),
$$

where for $T_{k} \leq n_{k}$ and $t_{k}=1, \ldots, n_{k}$,

$$
\xi_{k}\left(\left\{C_{j}^{k}\right\}_{j=1}^{t_{k-1}} \wedge D\right)=\sum_{j=1}^{t_{k}-1} R_{k} d_{k j}\left(\left\{C_{i}^{k}\right\}_{i=1}^{j-1}\right)+R R_{k} d_{k_{k}}\left(\left\{C_{i}^{k}\right\}_{i=1}^{t_{k}-1}\right)
$$

Under Assumption 2, the probability in (9) is

$$
P\left(\left\{X_{j}^{k}=C_{j}^{k}\right\}_{j=1}^{t_{k}-1} \cap\left\{X_{t_{k}}^{k}=D\right\}\right)=P\left(X_{t_{k}}^{k}=D \mid X_{t_{k}-1}^{k}=C_{t_{k}-1}^{k}\right) \cdot \prod_{j=1}^{t_{k}-2} P\left(X_{j+1}^{k}=C_{j+1}^{k} \mid X_{j}^{k}=C_{j}^{k}\right) P\left(X_{1}^{k}=C_{1}^{k}\right) .
$$

Let $\rho_{k}$ denote the marginal second moment of the random variable of year-ahead market value of unit capital invested in the $k$-th risky asset. Similar to the calculation of the expectation, it is

$$
\begin{aligned}
& \rho_{k}=E\left(\xi_{k}^{2}\left(\left\{X_{j}^{k}\right\}_{j=1}^{\min \left\{T_{k}, n_{k}\right\}}\right)\right)=\sum_{C_{1}^{k}, \ldots, C_{n_{k}}^{k} \in S \backslash\{D\}} \xi_{k}^{2}\left(\left\{C_{j}^{k}\right\}_{j=1}^{n_{k}}\right) \prod_{j=1}^{n_{k}-1} P\left(X_{j+1}^{k}=C_{j+1}^{k} \mid X_{j}^{k}=C_{j}^{k}\right) P\left(X_{1}^{k}=C_{1}^{k}\right) \\
& +\sum_{t_{k}=1}^{n_{k}} \sum_{C_{1}^{k}, \ldots, C_{t_{k}-1}^{k} \in S \backslash\{D\}} \xi_{k}^{2}\left(\left\{C_{j}^{k}\right\}_{j=1}^{t_{k}-1} \wedge D\right) P\left(X_{t_{k}}^{k}=D \mid X_{t_{k}-1}^{k}=C_{t_{k}-1}^{k}\right) \prod_{j=1}^{t_{k}-2} P\left(X_{j+1}^{k}=C_{j+1}^{k} \mid X_{j}^{k}=C_{j}^{k}\right) P\left(X_{1}^{k}=C_{1}^{k}\right)
\end{aligned}
$$

\section{The distributionally robust chance constrained asset allocation model}

A classical distributionally robust chance constrained optimization model can be specified as follows:

$$
\begin{gathered}
\text { minimize }_{x \in \chi} \quad c^{\mathrm{T}} x \\
\text { subject to } \min _{F \in \mathscr{D}} P_{F}\left(y_{0}(x)+y(x)^{T} \xi \leq 0\right) \geq 1-\varepsilon,
\end{gathered}
$$

where $x$ is the decision vector, for example, investment proportions in our study; $c$ is the cost vector, i.e. a vector of deterministic parameters; $y_{0}(x)$ and $y(x)$ are linear functions of $x$; $\xi$ is a vector of uncertainty parameters and in this study it denotes a future mark-to-market value vector of the risky assets, which is highly correlated with the credit rating migration; $F$ is a possible distribution of uncertainty parameters $\xi$; $\mathscr{D}$ is a set of probability distributions, assumed to include the true distribution of $\xi$ and is called uncertainty set. The inequality in (13) is referred to as distributionally robust chance constraint which implies the constraint " $y_{0}(x)+$ $y(x)^{\mathrm{T}} \xi \leq 0$ " is satisfied with a sufficiently high probability (safety probability) i.e. greater than or equal to 1- $\varepsilon$, with respect to the worst-case over the choice of a distribution. Three values of $1-\varepsilon$ are commonly considered, which are 0.90, 0.95 and 0.99 (Rockafellar and Uryasev 2000), to reflect different risk attitude. In this study, " $y_{0}(x)+y(x)^{\mathrm{T}} \xi \leq 0$ " is a reformulated form for the CAR requirement, for example, "CAR $\geq 10.5 \%$ ", as CAR is a function of $x$ and $\xi$.

The conventional distributionally robust chance constrained models are formulated and solved based on the first and the second moment of $\xi$ (El Ghaoui et al 2003; Calafiore and EI Ghaoui 2006; Zymler et al. 2013A, 2013B; Sun et al. 2017). Following the spirit of Lu et al. (2015), our model is based on only the first-order and the marginal second-order moment, rather than the covariance matrix of $\xi$, so our method is immune to the estimation errors of the covariance of $\xi$.

In this section, we construct the distributionally robust asset portfolio optimization model. In the optimization model, the objective function is maximizing the expected rate of return, subject to a CAR chance constraint. This CAR chance constraint is to guarantee a bank's capital meet the authority regulatory requirement.

\subsection{Objective function}

Let the $(n+l) \times 1$ vector $x=\left(x_{1}, \ldots, x_{n}, x_{n+1}, \ldots, x_{n+l}\right)^{\mathrm{T}}$ denote the assets allocation, where the first $n$ variables 
represent risky assets' proportions and the latter $l$ ones represent risk-free assets' proportions. Let $R=\left(R_{1}, \ldots, R_{n}\right.$, $\left.R_{n+1}, \ldots, \mathrm{R}_{n+l}\right)^{\mathrm{T}}$ be the $(n+l) \times 1$ vector of the assets' expected one-year-ahead rate of return. To maximize the bank asset portfolio rate of return, the objective function in our model is

$$
\text { maximize }{ }_{x \in \chi} R^{\mathrm{T}} \text {, }
$$

where $x$ is the decision vector, and $\chi \subseteq R^{n+l}$ is a set of feasible solutions, which is a convex closed set. In this paper, the risky asset expected one-year-ahead return is estimated as the probability-weighted average of the return under each possible one-year-ahead credit rating. The validation of this estimation method relies on the assumption that each single loan's credit rating process follows a time-homogenous Markov chain, i.e. Assumption 2 in 2.3,

\subsection{The CAR-based chance constraint}

Let $T A$ denote the total asset of a bank and $T L$ be the total liability, which are known constants. $\zeta^{\mathrm{T}}=\left(\xi^{\mathrm{T}} ; \gamma^{\mathrm{T}}\right)$ denotes the vector of one-year-ahead market value of one unit capital invested in each asset, where $\xi=\left(\xi_{1}, \ldots, \xi_{n}\right)^{\mathrm{T}}$ is a vector of uncertainty parameters and $\gamma=\left(\gamma_{1}, \ldots, \gamma_{l}\right)^{\mathrm{T}}$ is a vector of deterministic parameters. In this study, we assume that credit rating migration does not occur for risk-free assets. Therefore, one unit capital invested to the $k$-th risk-free asset is worth the sum of one unit capital (principle) and its annual interest at the 1-year-end. Let $w_{k}$ represent the $k$-th asset's weight factor depending on its risk rating. Let $r$ be the target CAR of a bank. Normally, $r$ is set by the bank's stakeholders and equal to $8 \%+\delta$, where $\delta$ is the capital conservation buffer representing the additional CAR exceeding the lowest CAR requirement of $8 \%$ based on Basel II (Basel Committee 2004).

The CAR chance constraint is constructed as follows

$$
\text { minimize }_{F \in \mathscr{D}} P_{F}\left(\frac{T A \zeta^{T} x-L T}{T A\left(w_{1} \xi_{1} x_{1}+\cdots+w_{n} \xi_{n} x_{n}+w_{n+1} \gamma_{1} x_{n+1}+\cdots+w_{n+l} \gamma_{l} x_{n+l}\right)} \geq r\right) \geq 1-\varepsilon,
$$

where $F$ is the probability distribution of $\xi$, i.e. the one-year-ahead market value of $n$ risky assets; $\mathscr{D}$ is a set of probability distribution that is assumed to include the true distribution $F ; P_{F}(\bullet)$ is probability value computed with respecte to random vector $\xi$ given that it follows the probability distribution $F$; TA $\zeta^{T} x-L T$ represents the equity worth; and $T A\left(w_{1} \xi_{1} x_{1}+\cdots+w_{n+l} \gamma\left(x_{n+l}\right)\right.$ is the total risk-weighted assets at the 1-year-end. As proposed by Basel Committee, a bank's CAR is defined as the quotient in the parentheses of (15). In this CAR chance constraint, the safety probability $(1-\varepsilon)$ is determined ex ante by the assets managers of a bank reflecting his/her risk attitude. In particular, higher safety probability reflect stronger risk adverse attitude.

As discussed earlier, in practice limited information on $F$, i.e. the distribution of the uncertain parameters $\xi$, is available for the decision making process. The full second order moment of $\xi$ is hard to estimate precisely (Bucay and Rosen 1999; Pu and Zhao 2012). However it is more convenient and precise to estimate the first order and the marginal second order moment of the random vector $\xi$. Therefore, in this study, $\mathscr{D}$ is defined as:

$$
\mathscr{D}\left(R^{n}, \mu, \rho\right)=\left\{F \in \Xi: P\left(\xi \in R^{n}\right)=1, E_{F}\left[\xi_{k}\right]=\mu_{k}, E_{F}\left[\xi_{k}^{2}\right]=\rho_{k}, k=1,2, \ldots, n\right\},
$$

where $R^{n}$ is $n$-dimensional real number space and contains the support of $F ; \Xi$ is the set of all possible probability measures on $R^{n} ; E_{F}(\bullet)$ is the expectation of $\bullet$ given that it follows the distribution $F ; \mu_{k}$ and $\rho_{k}$ are the first and second moment of one-year-ahead market value of the $k$-th loan, respectively (see Equations (5) and (12)). In this paper, $\mathscr{D}\left(R^{n}, \mu, \rho\right)$ is called uncertainty set and assumed to be closed convex set, indicated by the shorthand notation of $\mathscr{D}$.

The combination of the CAR chance constraint (15) and the uncertainty set (16) guarantees even under the worst case of future market value of asset portfolio in the uncertainty set of $\mathscr{D}$, a bank is able to reach the regulatory target of CAR with a probability greater than the safety probability. Given the future market values of risk-free assets, i.e. $\gamma$, are deterministic, taking $\gamma^{T}=\left(1+R_{n+1}, \ldots, 1+R_{n+l}\right)$ into the left hand side of inequality 
(15), our chance constraint can be transformed into the constraint of a classical distributionally robust chance constrained optimization model as follows ${ }^{1}$ :

$$
\min _{F \in \mathscr{D}} P_{F}\left(y_{0}(x)+y(x)^{T} \xi \leq 0\right) \geq 1-\varepsilon,
$$

where

$$
\begin{gathered}
y_{0}(x)=T L-T A \sum_{k=n+1}^{n+l}\left(1+R_{k}\right) g_{k} x_{k}, \\
y(x)^{T}=-T A\left(g_{1} x_{1}, \ldots, g_{n} x_{n}\right), \\
g_{k}=1-r w_{k}, k=1,2, \ldots, n+l .
\end{gathered}
$$

It can be seen that $y_{0}(x)$ and $y(x)$ are linear functions in $x$.

The chance-constrained program under the assumption that the random parameters' complete probability distribution $F$ is known was first discussed by Charnes and Cooper (1959) and Miller and Wagner (1965). Because of the difficulty to obtain the full and accurate information of the probability distribution of the random parameters, the distributionally robust approach, which relies only on partial information of the probability distribution, is widely adopted recently. For example, Calafiore and EI Ghaoui (2006) and Zymler et al. (2013A, 2013B) use the mean and covariance matrix of the uncertainty parameters to construct the uncertainty set $\mathscr{D}$, which is commonly adopted in the previous studies. However this method is not applicable to our study, because the joint credit migrations and market values of two risky assets are not directly observable. To solve this problem, the previous studies (see Bucay and Rosen 1999; Hilscher and Wilson 2017) use an observable financial factor, such as equity correlations of publicly traded firms, as a proxy for asset correlations. This approach may lead to inaccurate estimation on the covariance of risky assets' future market values, which will cause poor decision making based on the optimization model using these inaccurate estimations (Delage and Ye 2010; Dorfleitner and Pfister 2013). In this study, we only used marginal moment information of a single asset to construct the uncertainty set (which are straightforward to observe and estimate), so that we can avoid the negative impact of the inaccurate estimation on the probability information.

Next we show that the inequality (17), which is the distributionally chance constraint of the optimization problem (14), has a tractable representation in terms of bilinear matrix inequalities.

Proposition 1. For any given $x \in \chi$, the inequality (17) can be equivalently transformed into the following system of inequalities:

$$
\begin{gathered}
\theta+\alpha^{\mathrm{T}} \mu+\beta^{\mathrm{T}} \rho \geq 1-\varepsilon, \\
{\left[\begin{array}{cc}
\theta-1 & \frac{1}{2} \alpha^{T} \\
\frac{1}{2} \alpha & \operatorname{Diag}(\beta)
\end{array}\right] \prec 0,} \\
{\left[\begin{array}{cc}
\theta+\lambda y_{0}(x) & \frac{1}{2}(\alpha+\lambda y(x))^{T} \\
\frac{1}{2}(\alpha+\lambda y(x)) & \operatorname{Diag}(\beta)
\end{array}\right] \prec 0,} \\
\theta \in R, \alpha \in R^{n}, \beta \in R^{n} \\
\lambda \geq 0,
\end{gathered}
$$

${ }^{1}$ For brevity, "maximize" and "minimize" are replaced by "max" and "min" in the rest of the paper. 
where $\mu^{T}=\left(\mu_{1}, \ldots, \mu_{n}\right)$ denotes the one-year-ahead expected market value vector of $n$ risky assets; $\rho^{T}=\left(\rho_{1}, \ldots, \rho_{n}\right)$ denotes the marginal second-order moment vector of one-year-ahead market value of $n$ risky assets; $\theta, \alpha$, $\beta$, and $\lambda$ are the lagrangian multipliers ( $\theta$ and $\lambda$ are real numbers; and $\alpha, \beta$ are $n \times 1$ vectors); $y_{0}(x)$ and $y(x)$ are denoted as (18a) and (18b); and " $<0$ " stands for negative semi-definite.

So far, the distributionally robust chance constraint (17) of the assets allocation model (14)-(16) has been transformed into (19a)-(19e) by applying the duality theory in infinite-dimensional optimization problem and the theory of conic linear moment problems (see Rockafellar 1970 and Shapiro 2001 for more details). The transformation details and the proof of Proposition 1 are presented in Appendix A.

\subsection{Other constraints}

Besides the CAR constraint, we impose the ratio of risky asset to total asset constraint, the investment diversification constraint and the capital budget constraint on the asset allocation model to reduce the investment risk. They are presented, respectively, as follows

$$
\begin{gathered}
\sum_{i=1}^{n} x_{i} \leq a, \\
l_{i} \leq x_{i} \leq u_{i}, i=1,2, \ldots, n+l, \\
\quad \mathbf{I}^{T} x=1 .
\end{gathered}
$$

In the constraints above (20a)-(20c), $x$ is the decision variable, representing the investment proportions. $a$ is a pre-determined number between 0 and 1, representing the maximum proportion of total asset invested in risky assets. The value of $a$ depends on an investor's preference. However, in common practice, $a$ is a relatively large value, because a bank relies on risky assets to generate higher return. For example, in our simulation study, $a$ is set to be $75 \%$, i.e. the total investment proportion of risky assets is constrained to be no more than $75 \%$ of the portfolio value. Meanwhile, this constraint implies that the total investment proportion of risk-free assets is at least $25 \% . l_{i}$ and $u_{i}$ are the minimum and maximum proportions that the $i$-th asset is allowed to take on. They are real numbers between 0 and 1, which implies that selling assets is not applicable in this study. $\mathbf{I}$ is a vector with all components of 1 . In practice, to assure investment diversity, $u_{i}(i=1,2, \ldots, n)$, i.e. the upper limitation on the investment proportion of a risky asset, is relatively small. For example, in our study, $u_{i}=0.2, i=1,2, \ldots, n$, indicating that a risky asset's investment proportion cannot exceed $20 \%$. In contrast, the diversity constraint can be much weaker for a risk-free asset, like in our study some $u_{i}=1(i=n+1, n+2, \ldots, n+l)$. With respect to the linear constraints (20a)-(20c), a feasible solution is available if there is at least one risk-free asset having the possible maximum investment proportion as 1 (i.e. its $u_{i}$ is 1 ), conditional on that there is no asset to be sold (i.e. $l_{i} \geq 0$ ).

\subsection{The asset allocation model and its solution}

The main result of this study is as follows.

Theorem 1.The robust bank asset allocation problem with the objective function (14) and CAR chance constraint (15) and structure restrictions (20) can be reformulated as the following linear semi-definite programming (SDP) model:

$$
\max _{x, \theta^{*}, \alpha^{*}, \beta^{*}, \lambda^{*}>0} R^{T} x,
$$

Subject to $\quad \theta^{*}+\alpha^{* \mathrm{~T}} \mu+\beta^{* \mathrm{~T}} \rho-(1-\varepsilon) \lambda^{*} \geq 0$, 


$$
\begin{gathered}
{\left[\begin{array}{cc}
\theta^{*}-\lambda^{*} & \frac{1}{2} \alpha^{* T} \\
\frac{1}{2} \alpha^{*} & \operatorname{Diag}\left(\beta^{*}\right)
\end{array}\right] \prec 0,} \\
{\left[\begin{array}{cc}
\theta^{*}+y_{0}(x) \quad \frac{1}{2}\left(\alpha^{*}+y(x)\right)^{T} \\
\frac{1}{2}\left(\alpha^{*}+y(x)\right) \quad \operatorname{Diag}\left(\beta^{*}\right)
\end{array}\right] \prec 0,} \\
\theta^{*} \in R, \alpha^{*} \in R^{n}, \beta^{*} \in R^{n} \\
\sum_{i=1}^{n} x_{i} \leq a, \\
l_{i} \leq x_{i} \leq u_{i}, i=1,2, \ldots, n+l, \\
\mathrm{I}^{T} x=1,
\end{gathered}
$$

The above linear semi-definite programming (SDP) model can be solved by well-developed polynomial-time interior-point methods. In our implementation, we adopt a widely used SDP solver, i.e. the CVX solver (Grant et al. 2008), which is called either Sedumi (Sturm 1999) or SDPT3 (Toh et al. 2012). Proof of Theorem 1 is presented in Appendix B.

\subsection{Extensions}

The Basel Committee on Banking Supervision (BCBS) urges all banks to move risk measure from VaR to expected shortfall, i.e., CVaR, because $\mathrm{CVaR}$ can better capture "tail risk" (see Bank for International Settlements 2012, 2013). This policy implementation may make CVaR become a standard risk measurement for financial industry in the near future. Therefore optimization model based on CVaR for asset allocation problem and regulatory capital planning problem will increasingly receive more attention from both regulators and bank managers. In fact, our method can be extended to solve the worst-case CVaR constrained optimization problem with marginal second-order moment information, for example, the credit CVaR optimization problem.

The worst-case CVaR constraint, with full second moment information, can be written as

$$
\sup _{F \in \mathbb{P}} P-C \operatorname{VaR}_{\varepsilon}\left(y_{0}(x)+y(x)^{T} \xi\right) \leq 0,
$$

and the distributionally robust chance constraint with the full second moment information can be written as

$$
\inf _{F \in \mathbb{P}} P\left(y_{0}(x)+y(x)^{T} \xi \leq 0\right) \geq 1-\varepsilon,
$$

where $\mathbb{P}$ is defined as the uncertainty set of all probability distributions of the random parameter $\xi$. Zymler et al. (2013B) show the equivalence between these two constraints. As shown in the previous discussion, our paper provides a way to reformulate a distributionally robust chance constrained optimization model to a tractable linear semi-definite programming model.

If the full second moment information is not available, the worst-case CVaR constraint with marginal second moment information can be constructed. Following the spirit of Zymler et al. (2013B), if the worst-Case $\mathrm{CVaR}$ constraint is equivalent to the distributionally robust chance constraint with marginal second-order moment information, by following our study, the worst-Case CVaR constrained optimization problem with 
marginal second-order moment information can be eventually reformulated to a tractable linear semi-definite programming problem. This can be applied to different type of portfolio selection problems when full covariance information is not available.

\section{Numerical simulations and results}

In this section we conduct a simulation study to apply our model to solve asset allocation problems for a hypothetical commercial bank. We assume the bank is in the US and named ABC Bank. We consider the asset allocation problems in two years of 2007 and 2013, i.e. an economic downturn and an economic upturn, respectively. In the simulation study, we also examine how some important factors, including minimum target CAR, safety probability and recovery rate of risky assets, impact on the optimal asset allocation.

\subsection{Simulation designs}

First of all, we consider an asset allocation problem for the ABC bank in the beginning of year 2007. There are two reasons to choose year 2007. First, 2007-2010 is a financial and economic crisis period in the US, i.e. an economic downturn. Second, in 2004 the BCBS suggested to implement the Basel II, a revised accord on capital regulation, in 2007. This regulation was expected to implement in 2008 in the US (see Heid 2007). In this simulation study, we examine the performance of our model in an economic downturn on assuring the regulatory capital requirement can be met. We, then, change the context to year 2013, i.e. an economic upturn in the US. For the purpose of model performance comparison, we consider the same asset portfolio.

In particular, we consider an asset portfolio which consists of 13 hypothetical assets ${ }^{2}$, including 12 fixed-rate commercial loans and 1 risk-free asset. The 12 commercial loans are various common loan types in practice, for examples, project financing, industrial loan, retail loan, and so on. The loan characteristics, including maturity, initiate credit rating, collateral type, recovery rate, annual interest rate, risk weight, the first-order and marginal second-order moments of one-year-ahead mark-to-market value of each risky loan are presented in Panel A of Table 4 and Table 5 for year 2007 and 2013, respectively. They are all input parameters of our model. In particular, the maturities, initiate credit ratings and collateral types are hypothetical, because the loan agreement between a borrower and a commercial bank is normally not publicly available. These loan characteristics remain the same in the second scenario of year 2013.

In this study, we set the recovery rate of each risky loan, following the spirit of Khieu et al. (2012). According to Khieu et al. (2012), recovery rate is determined by loan characteristics, recovery process characteristics, firm characteristics, macroeconomic conditions, industry characteristics and probability of default. Using Moody's Ultimate Recovery Database, Khieu et al. (2012) find the average recovery rate of bank loans over year 1987 to 2007 is $84.14 \%$ and the standard deviation is $28.22 \%$. First, we set the recovery rate of a loan with long maturity (longer or equal to 5 years) and no collateral in year 2007 as 55\% (about one standard deviation below the average recovery rate). It is the minimum recovery rate in our simulation study, as longer maturity, poor quality of collateral and downturn economy have negative impact on recovery rate. Therefore, we give loans with shorter maturity and better collateral higher recovery rates. Details are presented in Table 6. We set the recovery rates of the 2013 loans 5\% higher than their 2007 corresponding loans' recovery rates because of the improvement of macroeconomic condition in 2013.

The annual interest rate is varying across loans and not publicly available too. We first obtain the

\footnotetext{
${ }^{2}$ In the simulation studies, for simplicity, we only consider 13 assets. We acknowledge that a real bank asset allocation problem may involve a large number of assets. To avoid large-scale problem, those assets sharing common characteristics can be combined into a particular asset group before applying our model. The large-scale asset allocation problems have been considered in the literature (Sirigano et al. 2016), but it is out of the scope of this study.
} 
benchmark of interest rate from the survey of terms of business lending, released by the Board of Governors of Federal Reserve System in 2007. Based on this benchmark, we assign a hypothetical interest rate to each loan according to its loan term and initial credit rating. The interest rate of a loan in 2013 is set to be lower than its corresponding loan's interest rate in 2007 due to the upturn economy of 2013. As for the risk weight of each loan, we assume the ABC Bank implements the standardized approach of capital adequacy ratio-Basel $\mathrm{II}^{3}$ and assigns specific risk weights to the borrowers accordingly. We assume the risk weights of all risky loans are the same across the two years of 2007 and 2013.

The risk-free asset is a 1-year treasury bill (T-bill). The annual interest rate is set to be $3.50 \%$ and $0.13 \%$, in 2007 and 2013, respectively. We assume that risk-free asset will not default; therefore the recovery rate is set to be $100 \%$. The risk weight for the risk-free assets is $0 \%$, according to the standardized approach of capital adequacy ratio-Basel II. Recovery rate and risk weight remain the same over the two years. This information is shown in Panel B of Table 4 and Table 5.

In Table 4 and 5, we also present the first-order and marginal second-order moments of one-year-ahead mark-to-market value for each asset. We take them as known input parameters for our model. For the 12 risky assets, the moments are calculated by using Equations (5) and (12). For the 1-year T-bill, the expectation of one-year-ahead market value of unit capital invested is $1+r$, i.e. principle plus annual interest rate, and the standard deviation is 0 , given it is a risk free asset and will not default.

In addition, in 2007 the $\mathrm{ABC}$ bank's total asset and liability value are set as 1,562,147 million and 1,438,926 million US dollars, respectively, which are another set of input parameters for our model. In fact, J.P. Morgan Chase \&Co in 2007 had the same values for total asset and liability, as reported by the Bank scope Database. We follow the same practice to use the corresponding values of J.P. Morgan Chase \&Co in 2013 as the ABC Bank's total asset and liability value in 2013. They are 2,415,689 million and 2,204,511 million US dollars, respectively.

In these two simulation scenarios, we first set the target CAR as $10.5 \%$ and the safety probability as $99 \%$. We conduct a range of sensitivity tests to examine the impacts of these pre-determined parameters' on asset allocation decision, and the results are discussed in details in the subsection of 4.2.1 and 4.2.2. The ratio of risky asset to total asset constraint is set as $x_{1}+x_{2}+\cdots+x_{12} \leq 75 \%$. The investment diversification constraint is as 0 $\leq x_{i} \leq 0.2(i=1,2, \ldots, 12)$, and $0.25 \leq x_{13} \leq 1$. The positive investment constraint excludes a short-selling, as it is commonly unrealistic in a loan portfolio setting. Following Andersson et al. (2001), we set the upper trading limit of each risky loan as 0.2 , indicating that each loan's investment proportion cannot exceed $20 \%$ of the whole portfolio. And the capital budget constraint is $x_{1}+x_{2}+\cdots+x_{13}=1$.

\subsection{Results}

The optimal asset allocations for the above two scenarios are presented in Table 7. In general, our model suggests different optimal allocations under different economic conditions. In particular, the optimal investment strategy in 2013 , i.e. during an economic upturn, place a higher proportion of investment on high-risk and high-return loans, comparing to that in 2007, which is an economic downturn. More specifically, investment proportions of 4 risky loans have been changed. The investment proportion of loan 5, a low return loan with interest rate of $7.37 \%$ in 2007 , decreases significantly from 0.0521 in 2007 to 0 in 2013 . On the opposite, the investment proportions of two high-return loans, i.e. loan 4 and 8 (with interest rate of $7.99 \%$ and $7.90 \%$, respectively, in 2007), increase to 0.0746 and 0.0754 , respectively, in 2013 from 0 in 2007.

However it is interesting to see that the investment proportion of loan 3, a high-return loan with interest

\footnotetext{
${ }^{3}$ It is not necessary for a bank to use the Basel II standardized method to calculate its CAR. There are other methods used in practice to calculate the $\mathrm{CAR}$, such as the internal rating based approach. In this study, we assume the $\mathrm{ABC}$ bank adopts the standardized method, and all our conclusions are based on this assumption too.
} 
rate of $7.89 \%$ in 2007, decreases from 0.0979 in 2007 to 0 in 2013. A possible reason for this change is the trade-off between risk and return of the overall loan portfolio. The investment proportions of the other 8 risky loans and the 1 risk-free assets remain the same in 2013 comparing with those of 2007.

In 2007, the optimal asset allocation, suggested by our model, brings $6.7394 \%$ overall rate of return to the ABC bank (shown in Table 7, indicated by RoR i.e. overall portfolio rate of return). In 2013, the overall rate of return from the optimal asset allocation reduces to $3.7155 \%$, due to the individual interest rates are generally lower than 2007. However if the same 2007 asset allocation was adopted again in 2013, the overall rate of return would be $3.6773 \%$; about $0.0382 \%$ lower than that from the optimal allocation. In 2013, during an economic booming time, the optimal investment strategy suggested by our model is more aggressive to invest more in the high-risk and high-return assets, but assures that regulatory requirement of capital adequacy ratio is met.

At this point, it is worthwhile to acknowledge that the expected rate of return is notoriously difficult to estimate in practice. A considerable amount of effort has been devoted to estimate it more precisely. Generally speaking, there are two lines of research in this area. Along the first line, a range of models have been proposed to estimate the expected return, including, parametric estimation method under assumption that asset returns follow some specific distributional form (for static time case, see Andersson et al. 2001; Mencía, 2012) or stochastic process (for dynamic time case see Sirignano et al. 2016; Sirignano and Giesecke 2018; Alti and Titman forthcoming) and non-parametric estimation method (Yao et al. 2015). In this study, we adopt a parametric estimation method as explained in Section 2. The second line of research is focused on the parameter calibration. For example, in this study as we take into account of the credit migration risk, the expected rate of return of a risky loan depends on two parameters, i.e. probability of default and recovery rate (or loss given default). Along the second line, an extensive literature has identified a large number of factors influencing these two parameters, such as firm-idiosyncratic characteristics (Westgaard and Van Der Wijst 2001; Khieu et al. 2012; Hilscher and Wilson 2017), loan-lever features (Beck et al. 2018; Sirignano and Giesecke 2018; Sariannidis et al. 2019), macroeconomic (or systematic) risk factors (Khieu et al. 2012; Betz et al. 2018; Sirignano and Giesecke 2018; Hilscher and Wilson 2017) and industry risk factor (James and Kizilaslan 2014). In this study, we take as many as possible of these factors into consideration.

The expected rate of return of each loan, estimated based on its default probability and recovery rate, is treated as an input of our asset allocation model. By feeding these inputs into our model, an optimal asset allocation, which maximizes the overall portfolio return, can be obtained. More comprehensive estimation methods for the expected rate of return can be applied to bring in more accurate inputs to our model. Research in this area is of interest, but out of the scope of the current study. In this study, we aim to examine the trend in the overall portfolio return, following changes in key factors, including the safety probability, CAR target, and recovery rate. As presented in the following sections, the result discussion and the conclusions obtained from these results are focused on these trends, but not the estimated magnitude.

\subsubsection{Sensitivity test - safety probability}

The safety probability is normally pre-determined by a bank manager and reflects his/her risk attitude, i.e. higher safety probability reflects more severe risk adverse attitude. In this study, we conduct a sensitivity analysis to investigate the impact of safety probability on the optimal asset allocations in year 2007 and 2013, respectively, by varying the safety probability from $90 \%$ to $99 \%$. All the other input parameters remain the same as in our simulation study, i.e. shown in Table 4 and Table 5. Results on the optimal proportions of capital invested in the 12 risky loans under each safety probability are presented in Table $\mathbf{8}$. The investment proportion of the risk-free asset does not change over different safety probabilities therefore we skip the corresponding results.

As shown in Table 8, investment flows from the high risk and high return loans to the low risk and low 
return ones when the safety probability increases. However, the optimal investment strategy maintains a certain amount of investment proportion in high-return loans to maximize the expected return when the safety probability level increases $(\leq 99 \%)$. Take the results for year 2007 as example, when the safety probability is low (i.e.90\% 93\%), the investment proportions in loans 3, 4 and 8 (i.e. the high-risk and high-return loans) keep the same at 0.2. The proportions change for two loans, i.e., loan 7 and loan 12. Both loans have an interest rate of $7.88 \%$, but the standard deviations of one-year-ahead market values are 0.0104 and 0.0253 , respectively. Money flows from loan 12 to loan 7, i.e. from a more risky loan to a relatively safer one, when safety probability level increases.

When safety probability is relatively high $(94 \% \sim 97 \%)$, the investment proportions of high-risk and high-return loans have been reduced in order to satisfy the bank's target CAR constraint. For example, in year 2007, loan 4 has the highest interest rate of 7.99\% and the highest standard deviation of one-year-ahead market value of 0.0678 . Its investment proportion is 0.2 if the safety probability is $95 \%$, and decreases to 0.1433 if the safety probability increases to $96 \%$. Meanwhile, loan 8 has the second highest interest of $7.90 \%$ and the second highest standard deviation of 0.0329 . Its investment proportion decreases from 0.0124 to 0.0067 if the safety probability increases $1 \%$ from $95 \%$. The investment proportion of 0.0624 has been squeezed out from loan 4 and loan 8 to loan 12. Comparing to loan 4 and loan 8 , loan 12 has lower return but lower risk as well.

When the safety probability is extremely high at $99 \%$, investment flows to the low-risk and low-return loans and almost has nothing left for the high-risk and high-return loans. In particular, the high-risk loan 4 and 8 won't be included in the optimal asset portfolio anymore. The only high-risk loan involved is loan 3 , but its investment proportion is reduced significantly from 0.2 when the safety probability is $97 \%$ to 0.0979 . The investment proportions of loan 7, loan 11, and loan 12, three middle-risk and middle-return loans, have reached their limits of 0.2. For the very first time, loan 5, a low-risk and low-return loan, is included in the optimal asset portfolio.

The sensitivity analysis shows that our model is sensitive to the safety probability, i.e. the optimal asset portfolio is different if safety probability is changed. Generally speaking, as the safety probability increases, investment is flowing from high-return and high-risk loans to middle-return and middle-risk loans, then to low-return and low-risk loans. However, the optimal investment strategy maintains a certain amount of investment proportion in high-return loans to maximize the expected return when the safety probability level increases $(\leq 99 \%)$. In fact, as shown by Figure 2, the overall return of portfolio is relatively stable. Taking year 2007 as example, when the safety probability is $90 \%$, the optimal overall return is $6.81 \%$. When the safety probability increases to an extremely high value of $99 \%$, the optimal overall return from our model is $6.74 \%$, only $0.07 \%$ lower than the former. Even though our model reacts to the change in safety probability sensitively, our model allocates an optimal amount of investment proportion in high-return loans to maximize the expected return rather than sacrifice it that much. The same trend can be seen from the results for year 2013. The investment strategies provided by our model comply with the Basel Capital Accord framework and maximize the investment returns, and take account of the bank managers' risk preferences. 

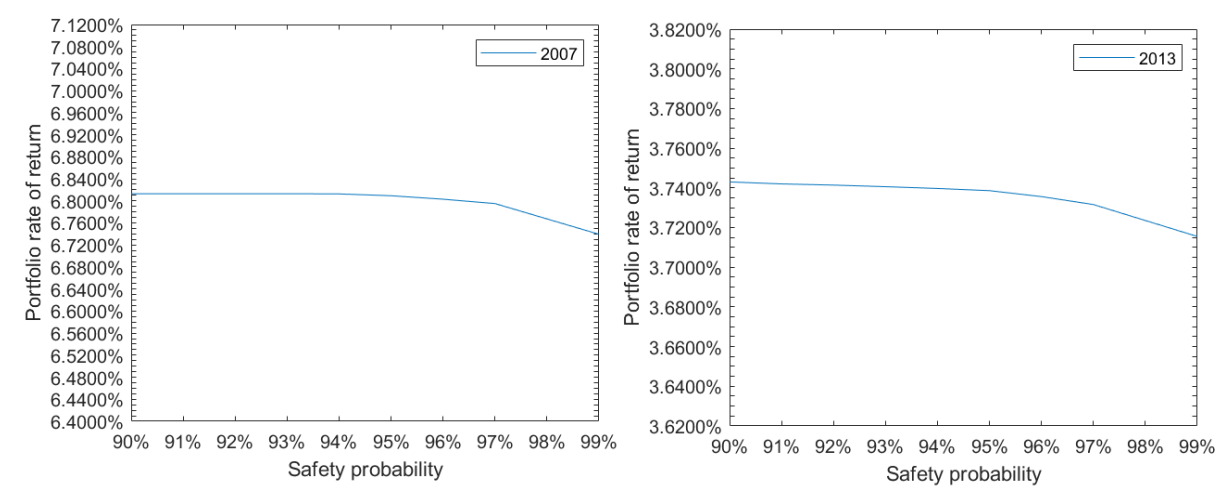

Fig.2 The overall rate of return of portfolio under different safety probabilities

\subsubsection{Sensitivity test - target CAR}

We conduct another sensitivity test to examine the effects of target CAR, increasing from $8 \%$ to $10.5 \%$, on the optimal asset allocation in year 2007 and 2013. In this analysis, we keep the safety probability the same as 95\%. The rest of the input parameters remain the same as in our simulation study, i.e. as presented in Table 4 and Table 5.The results on investment proportions of risky loans from this analysis are presented in Table 9. It is worthwhile to mention that the investment proportion of risk-free assets do not change, therefore the following discussion is focused on risky loans.

The results presented in Table 9 show that as the target CAR increases, the investment flows from high-risk and high-return loans to relatively low-risk and low-return loans. To some extent, the target CAR plays a similar role to the safety probability, i.e. both reflect the risk preferences of bank managers. More specifically, in 2007 the investment proportion of loan 8, a high-risk and high-return loan, decreases from 0.2 to 0.1565 when the target CAR increases from $8 \%$ to $9.5 \%$, and decreases further to 0.0124 when the target CAR increases to $10.5 \%$. On the opposite, the investment proportion of loan 12, a middle-risk and middle-return loan, increases from 0 to 0.1376 when target CAR increases from $9.5 \%$ to $10.5 \%$. Similar pattern can be seen in 2013.

In general, two results emerge from this sensitivity analysis. First, when target CAR is low, a bank is tend to invest in high-risk and high-return loans, i.e. loans with higher interest rate but relatively longer maturity, lower original rating and lower quality collateral, to gain relatively higher overall return, and vice versa. Second, in order to fulfill a higher CAR, the optimal asset allocation suggested by our model does not reduce the investment proportion in risky loans and assign more investment to risk-free assets. In fact, the investment proportion of risky loans has been kept the same at $75 \%$ with target CAR ranging from $8 \%$ to $10.5 \%$. Therefore, the expected return of portfolio is relatively stable with the increase of CAR, as shown by Figure 3. In particular, for year 2007, when CAR is $8 \%$ the overall return is $6.813 \%$ and when CAR increases to $10.5 \%$ the overall return is $6.809 \%$. This practice is different from the asset management strategy of Ito and Sasaki (2002) and Francis and Osborne (2012), who suggest banks with lower capitalization should slow down the investment in loans but invest more in risk-free asset (such as treasure bill) in order to reach the regulatory CAR. Our method provides an efficient way to deal with the trade-off between investment return and regulatory CAR. ${ }^{4}$

\footnotetext{
${ }^{4}$ This statement is valid, especially when CAR is calculated by the standardized approach. Further examination is required in order to obtain similar conclusion if CAR is calculated by other methods such as internal rating based approach.
} 

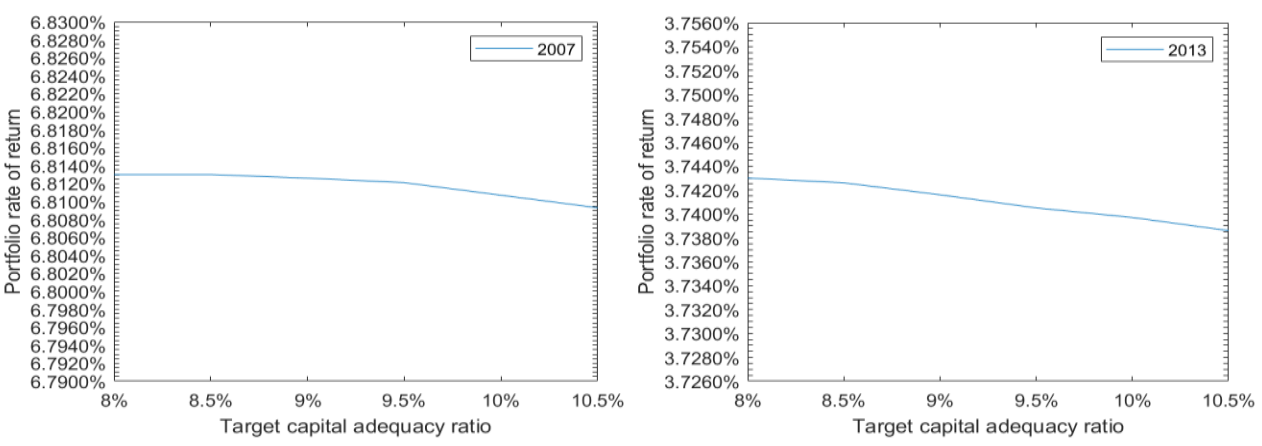

Fig.3 The overall rate of return of portfolio under different target CARs

\subsubsection{Sensitivity test- recovery rate}

In this study we also examine the impacts of recovery rates (RRs) of different types of loans on optimal asset allocations by using data from 2007. We choose four loans as representative loans, which are loan 4, loan 3 , loan 11, and loan 5 . We run a series of sensitivity tests, with each test having one loan's RR changing from $50 \%$ or $60 \%$ to $85 \%$ or $95 \%$, depending on the characteristics of the corresponding loan. In all sensitivity tests, we keep the target CAR at $10.5 \%$, the safety probability at either $95 \%$ or $99 \%$, and all the other input parameters remain the same as the main simulation study for year 2007.

Results on the optimal loan allocation and the corresponding overall rate of return are summarized in Table 10 and Table 11, for safety probability of 95\% and 99\%, respectively. Results on the risk-free asset allocation are not presented as they remain the same. We find that the impacts of RR on optimal asset portfolio allocation are different for different type of loans, when the safety probability is the same. However if the safety probability is different, the impacts of RR are different even for the same loan.

In particular, when the safety probability is $95 \%$, if we change the RR of loan 4 from $50 \%$ to $85 \%$, as shown in the upper left panel of Table 10, the optimal loan allocation has changed. First of all, the investment proportion of loan 4 increases from 0.1631 to 0.2 when its RR increases from $50 \%$ to $70 \%$, then keeps at 0.2 , i.e. the maximum investment proportion for a single loan in the portfolio. Second, the investment proportions of other loans have also changed. More specifically, the investment proportion of loan 8 increase from 0 to 0.2 when the RR of loan 4 increases from $50 \%$ to $70 \%$, meanwhile the total investment proportion of 3 relatively low-return (comparing to loan 4) loans, i.e. loan 3, loan 7 and loan 12, decreases by 0.2369 . When the RR of loan 4 continually increases to $80 \%$ and $85 \%$, the investment proportion of loan 7 , a middle-return loan, decreases further to 0.1424 and 0.1379 , respectively, however the proportions of the other two relatively high-return (comparing to loan 7) loans start to increase, i.e. proportion of loan 3 increases back to 0.2 and that of loan 12 increases first to 0.0076 then to 0.0121 . The expected overall rate of return is stable at around $6.8 \%$, with a slightly increasing trend when the RR of loan 4 increases.

Indicated by the results in the upper right panel of Table 10, the change in loan 3's RR has less impact on the optimal asset allocation, for example the high-risk and high-return loan 8 and the relatively low-return loan 7, than that in loan 4's RR. As shown in the lower panels of Table 10, the changes in RRs of loan 11 and loan 5, two middle (low)-risk and middle (low)-return loans, from $60 \%$ to $95 \%$ have no impact on the optimal asset allocation. Therefore, these changes do not cause any change in optimal return either.

When the safety probability is as high as $99 \%$, the results are very different, as shown in Table 11 . The changes in RRs of loan 4 (a super high-risk and high-return loan) has little impact on the optimal asset allocation. In particular, only when the RR of loan 4 increases from $50 \%$ to $70 \%$, the investment proportion in loan 3 increases from 0.0976 to 0.0978 and that in loan 5 decreases from 0.0524 to 0.0522 . There is no further change when RR of loan 4 keeps increasing to $80 \%$ or $85 \%$. One possible reason for the lack of impact from loan 4's RR change is the mixture of a super high safety requirement (i.e. 99\%) and super high risk level of 
loan 4. The changes in RRs of loan 3 (a high-risk and high-return loan), loan 11 (a middle-risk and middle-return loan) and loan 5 (a low-risk and low return loan) have more impacts on the optimal asset allocation when the safety probability is $99 \%$, compared to $95 \%$. These changes in RRs introduce changes in the optimal asset allocations. However the optimal returns is relatively stable.

This sensitivity analysis demonstrates that RR is a key factor in the decision making of investment allocation. The RR increase in high-risk and high-return loans normally has more significant impacts on asset allocation decision, than the increase in low-risk and low-return loans. This is particularly true when the safety probability is not as high as, for example, $99 \%$. However when the safety probability is super high, the changes in RR of those relatively low-risk and low-return loans will produce more change in asset allocation.

To further demonstrate the impact of RRs on optimal asset allocation, we conduct a series of extra simulation studies where we change the RRs of multiple loans simultaneously in the same direction, i.e. either increase or decrease different loans' RR. In particular, the results on the 12 risky loans are presented in Table 12 and 13. In the left panel of the two tables, we list the interest rate and recovery rate for each risky loan under the 2007 economy, which are the same with our main study design, as shown in Table 4. When the safety probability is $95 \%$, the results on the expected rate of return and asset allocation are presented in Table 12, i.e. the expected overall return is $6.8093 \%$. Based on this design, we change the recovery rate of loan 3 and 4 from $60 \%$ to $50 \%$, loan 7 from $85 \%$ to $60 \%$, and loan 8 and 12 from $80 \%$ to $60 \%$. Our model react to these changes by adjusting the optimal asset allocation. The overall return is reduced to $6.7925 \%$ as expected due to the poorer quality of several assets, however this has not brought dramatic negative impact on the overall return. We then changed the safety probability from $95 \%$ to $99 \%$. The results are presented in Table 13 . The left panel shows the optimal asset allocation and the expected overall return of $6.7394 \%$ when the recovery rates keep as the same with our main study design. We increase the recovery rate of loan 5, 11 and 12 from $80 \%$ to $95 \%$ and the right panel of Table 13 shows the corresponding results. The overall expected increases to $6.7727 \%$, which is also comparable to the results presented in Table 11 based on the same safety probability of $99 \%$. We can see that increasing the three loans' recovery rate causes the optimal asset allocation changed more and brings higher overall return as expected. From these simulation studies, we show that increasing (decreasing) RRs for multiple loans normally brings more changes in asset allocation strategy and expected overall return. Therefore in order to realize higher investment return under certain CAR requirement, a bank should encourage increase in RR of any loan, such as requesting better quality collateral.

\subsubsection{Comparison with CVaR model}

In the previous studies, the optimization model with minimum Conditional Value-at-Risk (CVaR) has been widely used to solve an asset allocation problem (see Rockafellar and Uryasev 2000; Andersson et al. 2001; Iscoe et al. 2012; Gao et al. 2017). The main difference between the CVaR model and our model is in the risk measure, i.e. the $\mathrm{CVaR}$ risk measure versus the capital adequacy ratio. As shown in Rockafellar and Uryasev (2000), the model can be expressed as follows:

$$
\min _{x \in R^{N}, \alpha \in R}\left\{F(x, \alpha)=\alpha+\frac{1}{1-\beta} \int_{y \in R^{M}}[f(x, y)-\alpha]^{+} p(y) d y: \sum_{i=1}^{N} x_{i} R_{i} \geq R^{*}, x_{i} \geq 0 \text { for } i=1, \ldots, N, \sum_{i=1}^{N} x_{i}=1\right\} .
$$

In problem (22), $x$ denotes the vector of asset allocation, and it is an output parameter of the model to be determined; $N$ denotes the number of assets in a portfolio; $\alpha$ is the Value-at-Risk (VaR) and it is another output parameter to be determined; $\beta$ is a input parameter and pre-determined by a decision-maker, indicating the level of confidence; $f(x, y)$ is the loss function and it is a random variable having a distribution in $R$ induced by the risky assets loss vector $\boldsymbol{y}$, which has a joint density function of $p(y) ; R_{i}(i=1, \ldots, N)$ is the $i$-th asset's rate of return; $[U]^{+}=U$, when $U>0$ otherwise $[U]^{+}=0$. In this optimization problem, the constraints include the overall expected return is higher than a given amount, i.e. $R^{*}$, the constraint that there is no asset to be sold and the capital 
budget constraint.

The analytical expression of problem (22) is difficult to derive for a general distribution of $p(y)$ (Nemirovski and Shapiro 2006), and a Monte Carlo (MC) simulation method is normally adopted to obtain an effective approximation for problem (22) (e.g. see Rockafellar and Uryasev 2000; Andersson et al. 2001; Iscoe et al. 2012). In this paper, for the purpose of comparison, we use a MC formulation of the CVaR minimization model subject to the expected return constraint (with $R^{*}=6.6 \%$ ), the investment diversification constraint and the capital budget constraint, with the latter two constrains have the same design with our main study. So the CVaR minimization model is as follows:

$$
\min _{x \in R^{13}, Z \in R^{J}, \alpha \in R}\left\{\begin{array}{l}
\varphi(x, Z, \alpha)=\alpha+\frac{1}{(1-\beta) J} \sum_{j=1}^{J} Z_{j}: Z_{j} \geq \sum_{i=1}^{12}\left(\left(b_{i}-y_{j i}\right) x_{i}\right)-\alpha, Z_{j} \geq 0, j=1, \ldots, J ; \\
\sum_{i=1}^{13} x_{i} R_{i} \geq R^{*} ; \sum_{i=1}^{12} x_{i} \leq 0.75 ; 0 \leq x_{i} \leq 0.2, i=1,2, \ldots, 12 ; 0.25 \leq x_{13} \leq 1 ; \sum_{i=1}^{13} x_{i}=1
\end{array}\right\},
$$

where $J$ denotes the number of all possible credit migrations scenarios; $b_{i}(i=1, \ldots, 13)$ denotes the one-year ahead value of the $i$-th asset with no credit migration so it is the sum of the principle and the interest; $y_{i j}$ $(i=1,2, \ldots, 13, j=1,2, \ldots, J)$ denotes the one-year ahead value of the $i$-th asset under the $j$-th credit migration scenario, which can be gained by the Credit-Metrics method as presented in Section 2.2; $\left(b_{i}-y_{i j}\right) x_{i}$ represents the loss of the $i$-th risky asset under the $j$-th credit migration scenario, and the sum over all assets, is an approximation of portfolio credit loss distribution under the $j$-th credit migration scenario; $Z_{j}(j=1, \ldots, J)$ are auxiliary variables and output parameters. According to our assumption, a risk-free asset's credit rating will not migrate, i.e. $b_{13}-y_{13 j}=0$ for any $j=1,2, \ldots, J$, therefore a risk-free asset's credit loss is not included in the approximation of the portfolio credit loss. The remaining parameters in (23) are defined the same with those in (22). The level of confidence, i.e. $\beta$, is set at $95 \%$ and 99\%, respectively. For simplicity, Assumption 2 in Section 2.3, i.e. each single loan's credit rating process follows a time-homogenous Markov chain, is hold here. We assume the actual one-year credit rating migrations of any two risky loans are independent, and the marginal one-year credit migration probabilities of a risky loan are consistent with the Standard \& Poor's transition matrix shown in Table 1. The overall approximation is fulfilled by a MC simulation with a certain sample size (i.e. the number of random selected credit migration scenarios).

In the first simulation study on the performance of CVaR model, we simulate 1000, 5000, 10,000 and 20,000 credit migration scenarios, according to the migration probability matrix (shown as Table 1) independently for each risky loan. That is to say, the simulation method we adopt for the overall approximation is consistent with the actual situation. The estimated investment proportions of the 12 risky loans by the CVaR model of different sample size, with confidence level of $95 \%$ and $99 \%$ are presented in Table 12 and Table 13, respectively. The optimal investment proportion of the risk-free asset, i.e. asset 13, keeps the same at 0.25 under different confidence level and sample size. We also calculate the expected portfolio return under different settings in terms of sample size and confidence level. The expected return is always $6.6 \%$, i.e. the threshold of the expected return constraint. In Table 12 and 13, we also report the corresponding CVaR from each optimal allocation and the computation time by using the linear optimization toolbox of Matlab 2016b on an Intel Core i5-7400@3.0 GHz processor. From these results, we can see that the optimal asset allocations suggested by the CVaR model vary corresponding to the sample size. It can be argued that larger sample size means a better approximation, therefore a better performance. However larger sample size also means longer computation time. For example, when sample size is 20,000 and confidence level, i.e. $\beta$, is 0.99 , the computation time for CVaR model is 3,137 seconds $^{5}$.

${ }^{5}$ It is worthwhile to notice that the calculation time of a CVaR problem has been speeded up, see for example, 
For the simulation with sample size of 20,000 and confidence level of $99 \%$, we calculate the CAR under each of the 20,000 credit migration scenarios. In this exercise, we keep the total asset value the same as in our simulation study for year 2007, i.e., 1,562,147 million US dollars, but the total liability $(T L)$ is varying at four values, which are 1,438,926, 1,463,570,1,488,214 and 1,512,858 million US dollars. Therefore, the ratio of total liability to total asset is approximately 92:100, 94:100, 95:100, and 97:100, respectively. In Table 14, we report the maximum, mean and minimum values of these 20,000 CAR under different $T L$ levels (or different ratios of $T L$ to $T A$ ), and we count the numbers of CARs which are less than 15\%,10.5\% and 8\%, respectively.

For the purpose of comparison, we apply our distributionally robust CAR chance constrained asset allocation model to the eight scenarios, i.e. with the four $T L$ levels stated above, two safety probability levels of $95 \%$ and $99 \%$, and minimum target capital adequacy ratio of $10.5 \%$. It is worthwhile to notice that the assumption of actual independent credit rating migrations of any two risky loans is irrelevant to our model, because our model only relies on the first-order and marginal second-order moment of one-year-ahead market value of a loan, but not the joint distribution. The results on investment proportions of the 12 risky loans, the expected optimal portfolio returns, and the computation times are presented in Table 15 and Table 16. The optimal investment proportion of the risk-free asset remains the same as 0.25 . The computation time of our model is always less than 1 second. It is interesting to see that the total liability level has a significant impact on the optimal asset allocation suggested by our model. Furthermore, the expected optimal portfolio return decreases when the total liability increases, i.e. the total net assets (as the total asset keeps the same) decreases. However the expected portfolio return gained from our model is always higher than that from the CVaR model, i.e. $6.6 \%$.

Following the same procedure, we calculate the corresponding results presented in Table 14 for our CAR model, which are presented in Table 17. Comparing the results in Table 14 and Table 17, we can see that the CARs, obtained from both the CVaR model and our model, are decreasing when the bank's liability level is increasing. However the average CARs from our model are always higher than those from the CVaR model at the same $T L$ level. Furthermore, none of the 20,000 CARs under the possible credit migrations from our model is lower than $10.5 \%$, i.e. the minimum target CAR. And 3 CARs from the CVaR model are lower than $10.5 \%$, i.e. violated the CAR regulation. This result is as expected, because the CVaR model aims to minimize the excess credit risk rather than, like our model, to assure the CAR regulatory requirement under all possible credit migration scenarios.

In the second simulation study on the performance of CVaR model, to approximate the overall CVaR, we adopt three different MC simulations with the same sample size of 20,000. Different from the previous simulation study, the simulation method we adopt for the overall approximation is inconsistent with the actual situation. In particular, in each MC simulation, 20,000 one-year-ahead asset values of the 12 risky loans are generated according to a given probability distribution, which is inconsistent with the actual distribution of the one-year-ahead asset values. The three probability distributions adopted by the MC simulation are two joint normal distributions and one independent uniform distribution. The details are presented below.

First, following the spirit of Rockafellar and Uryasev (2000), in the MC simulation we generate the 20,000 one-year-ahead values of the 12 risky loans, i.e. $\xi$, from a normal distribution of $N\left(\mu, \sum_{1}\right)$. Therefore the portfolio credit loss, i.e., the weighted average of each loan's credit loss of $\left(b_{i}-\xi_{i}\right) x_{i}$, follows a normal distribution too. In this MC simulation, we set $\mu$ as the 12 risky loans' estimated expected one-year-ahead values, presented in Panel A of Table 4. The diagonal components of covariance matrix $\sum_{1}$ are set to be the estimated variance of each loan's one-year-ahead value, i.e. the square of the estimated standard deviation of

Alexander et al. (2006). In this simulation study we adopt a conventional calculation method for the CVaR problem. The calculation time can be reduced if a more recent calculation method was applied. 
each loan as presented in Panel A of Table 4. We assign non-zero correlations, i.e. $\operatorname{corr}_{i, j}(i, j=1,2, \ldots, 12$ and $i \neq j$ ), to selected loans. In particular, $\operatorname{corr}_{1,3}=-0.62, \operatorname{corr}_{3,4}=0.4, \operatorname{corr}_{4,12}=0.8$ and the remaining correlations are set to be 0 . Obviously, this distribution of the uncertainty parameter $\xi$, i.e. $N\left(\mu, \Sigma_{1}\right)$, is in the distribution set $\mathscr{D}$, because the marginal moment conditions of $\xi$ are satisfied. We further set the level of confidence $\beta=99 \%$, and the the minimal overall expected return $R^{*}=6.6 \%$. The optimal allocation results of the 12 risky loans by this $\mathrm{CVaR}$ model are presented in the first row of Table 18. The risk-free asset's proposition is 0.25 .

Following the same practice to gain results presented in Table 14, we gain the corresponding results for the above CVaR model estimated by the MC simulation adopting $N\left(\mu, \sum_{1}\right)$ as the joint distribution of the 12 risky loans' one-year-ahead values. In particular, we first generate 20,000 credit rating migration scenarios according to the migration probability matrix (shown as Table 1) independently for each risky loan, i.e. the actual situation. We then calculate the CAR based on the optimal asset allocation suggested by the above CVaR model under each migration scenario. The results on minimum, mean and maximum CAR over the 20,000 scenarios at different $T L$ levels are presented in Table 19. Comparing to the results from the CVaR model estimated by the MC simulation adopting the true credit migration distribution (presented in Table 14), performance of this CVaR model, with biased distribution assumption, on CAR is worse. In general, the CARs gained from the miss-specified CVaR model are lower and more scenarios experience violation of CAR regulation.

Second, we adopt another normal distribution $N\left(\mu, \sum_{2}\right)$ in the $\mathrm{MC}$ simulation. The non-diagonal components of $\sum_{2}$ are different from $\sum_{1}$. In particular, $\operatorname{corr}_{1,3}=-0.3$, $\operatorname{corr}_{3,4}=0.4, \operatorname{corr}_{4,12}=0.9$. The remaining parameter values are the same as the ones in previous case, including $\mu, \beta, J, R^{*}$ and the "actual distribution" of the 12 loans' one-year-ahead values. The optimal allocation results of the 12 risky loans suggested by this CVaR model are presented in the second row of Table 18. The risk-free asset's proposition is 0.25 . The CAR performance from this simulation study is presented in Table 20. Similar conclusion can be seen.

Last, we adopt an independent uniform distribution in the MC simulation. Particularly, the $i$-th risky loan's one-year-ahead value $\xi_{i}(i=1,2, \ldots, 12)$ is simulated from an independent uniform distribution $\mathrm{U}\left(a_{i}, b_{i}\right)$. The parameters $a_{i}, b_{i}$ can be obtained via calculating $\left(a_{i}+b_{i}\right) / 2=\mu_{i}$ and $\left(b_{i}-a_{i}\right)^{2} / 12=\rho_{i}-\left(\mu_{i}\right)^{2}$, since expected value $\mu_{i}$ and variance $\rho_{i}-\left(\mu_{i}\right)^{2}$ of $\xi_{i}$ are known information as presented in Table 4. We then conduct the MC simulation to solve model (23) by sampling $y_{i j}$ from the independent uniform distributions $\mathrm{U}\left(a_{i}, b_{i}\right)$ and setting the remaining parameter values to be the same as the ones in previous cases. The optimal allocation results of the 12 risky loans estimated by this CVaR model are presented in the third row of Table 18 . The risk-free asset's proposition is 0.25 again. The corresponding CAR performance results are presented in Table 21, and similar conclusion can be obtained in this study.

In general, the optimal allocation results from the CVaR model are sensitive in terms of the joint distributions of uncertainty parameters adopted by the MC simulation to approximate the overall loss, especially when the adopted distribution by the MC simulation is different from the actual situation. However our model is immune from a biased distribution assumption. The CVaR model is not able to assure the CAR regulatory requirement under all possible credit migration scenarios, especially when the MC simulation adopts a miss-specified distribution for the uncertain parameters.

\section{Conclusion}

In this paper, we propose a robust bank asset allocation model by using a distributionally robust chance constrained optimization approach. The optimal asset allocation suggested by our model is able to not only take account of uncertain credit rating migration risk, but also assure the regulatory requirement on capital adequacy ratio is met. Our model is also superior to the conventional chance-constrained portfolio optimization method, which requires that the first-order and full second-order moments of one-year-ahead market value of unit capital invested are estimated to establish the chance constraint. But the full second moment, especially the 
covariance, of the one-year-ahead market value is normally not observable and hard to estimate accurately. Therefore a model based on an inaccurate estimation normally provides a biased portfolio decision-making. In this study, we adopt the Credit-Metrics method to compute the first moment and the marginal second moment (rather than the full second moment) of one-year-ahead market value of unit capital invested in a loan, in order to establish the chance constraint. Therefore our method is immune from the unavailability or the inaccurate estimation of covariance. By applying the duality theory in infinite-dimensional optimization problem and the theory of conic linear moment problems, we convert the intractable chance constraint optimization model into a tractable SDP problem.

We conduct a simulation study to illustrate the application of our model. A series of sensitivity tests is applied to examine the impacts of various factors, including safety probability, target CAR and recovery rate, on the optimal asset allocation decision and expected rate of return. The results show that during an economic downturn, more investment should be assigned to the relatively low-risk and low-return loans, while in the booming time, loans with higher return should be the main investment. When the safety probability or the target CAR increases, i.e. the bank is more risk adverse, the investment may flow from relatively high-risk and high-return loans to relatively low-risk and low-return ones. In terms of recovery rate, the change in one asset's recovery rate may lead to a change in the whole optimal portfolio, and the impact varies depending on the risk and return level of the corresponding asset. Our method can help bank managers to make a robust asset allocation decision, according to the asset portfolio's characteristics, CAR regulatory requirement, macroeconomic status, and bank managers' specific risk attitude, taking into account of the uncertainty in credit rate migration.

Furthermore, our method has another two merits. First, our model is more applicable than a distributional robust chance constrained optimization model using the marginal distribution of the uncertainty parameter, because the first and the marginal second moment of uncertainty parameter used in our method can be easily derived from the marginal distribution of uncertainty parameter. For example, if the marginal distribution of the future market value of risky assets such as loans is known for a commercial bank, which is compliant with the Basel III Accord, our model can be applied to make investment decision in an uncertainty environment. Second, our method can be extended to solve the worst-case CVaR constrained optimization model with marginal second-order moment information. This is especially relevant under the context that CVaR becomes more and more important in the banking and financial industries due to the promotion of CVaR, by Basel Committee on Banking Supervision, as a standard risk measure.

\section{Acknowledgments}

This work was supported in part by the NSFC under Grants 71671023, 71731003, and 71301017, in part by the Foundation for Innovative Research Groups of NSFC under Grant 71421001, in part by China Postdoctoral Science Foundation of Grant 2016M600207, the development Foundation of teaching and scientific research for teachers of Liberal Arts in Zhejiang University.

\section{Reference}

[1] Alexander, S., Coleman, T.F. \& Li, Y., (2006). Minimizing CVaR and VaR for a portfolio of derivatives. Journal of Banking \& Finance, 30(2), 583-605.

[2] Allen, D., Powell, R., \& Singh, A. (2016). Take it to the limit: Innovative CVaR applications to extreme credit risk measurement. European Journal of Operational Research, 249(2), 465-475.

[3] Alti, A. \& Titman, S. A Dynamic Model of Characteristic-Based Return Predictability. (Forthcoming). The Journal of Finance. 
[4] Altman, Edward I. (1998). The importance and subtlety of credit rating migration. Journal of Banking \& Finance, 22, 1231-1247.

[5] Altman, E. I. (1980). Commercial bank lending: process, credit scoring, and costs of errors in lending. Journal of Financial and Quantitative Analysis, 15(4): 813-832.

[6] Altman, E. I., \& Saunders, A. (1997). Credit risk measurement: developments over the last 20 years. Journal of banking \& finance, 21(11-12), 1721-1742.

[7] Altman, E. I., \& Saunders, A. (2001). An analysis and critique of the BIS proposal on capital adequacy and ratings. Journal of banking \& finance, 25(1), 25-46.

[8] Altman, E. I., Bharath, S. T., \& Saunders, A. (2002). Credit ratings and the BIS capital adequacy reform agenda. Journal of banking \& finance, 26(5), 909-921.

[9] Andersson, F., Mausser, H., Rosen, D., \& Uryasev, S. (2001). Credit risk optimization with conditional value-at-risk criterion. Mathematical Programming, 89(2), 273-291.

[10] Araten, M., Jacobs, M., \& Varshney, P. (2004). Measuring lgd on commercial loans: an 18-year internal study. RMA Journal, 86(8), 96-103.

[11] Artzner, P., Delbaen, F., Eber, J. M., \& Heath, D. (1999). Coherent measures of risk. Mathematical finance, 9(3), $203-228$.

[12] Bank for international settlements. (2012). Fundamental review of the trading book - consultative document. Available: http://www.bis.org/publ/bcbs219.htm.

[13] Bank for international settlements. (2013). Fundamental review of the trading book - second consultative document. Available: http://www.bis.org/publ/bcbs265.htm.

[14] Basel Committee. (2004). The international convergence of capital measurement and capital standards: a revised framework (Basel II). BIS, Basel.

[15] Beck, T., Ioannidou V., \& Schäfer, L. (2018). Foreigners vs. natives: bank lending technologies and loan pricing. Management Science, 64(8): 3792-3820.

[16] Betz, J., Kellner, R., \& Rösch, D. (2018). Systematic effects among loss given defaults and their implications on downturn estimation. European Journal of Operational Research, 271(3): 1113-1144.

[17] Bucay, N., \& Rosen, D. (1999). Credit risk of an international bond portfolio: a case study. ALGO Research Quarterly, 2(1), 9-29.

[18] Calafiore, G. C., \& El. Ghaoui, L. (2006). On distributionally robust chance-constrained linear programs. Journal of Optimization Theory and Applications, 130(1), 1-22.

[19] Charnes, A., \& Cooper, W. W. Chance-constrained programming. Management science, 1959, 6(1), 73-79.

[20] Das, S., Duffie, D., Kapadia, N., \& Saita, L. (2007). Common failings: how corporate defaults are correlated. The Journal of Finance, 62(1), 93-117.

[21] Delage, E., \& Ye, Y. (2010). Distributionally robust optimization under moment uncertainty with application to data-driven problems. Operations Research, 58(3), 595-612.

[22] Dorfleitner, G., \& Pfister, T. (2013). Capital allocation and per-unit risk in inhomogeneous and stressed credit portfolios. The Journal of Fixed Income, 22(3), 64-78.

[23] Duffie, D., Saita, L., \& Wang, K. (2007). Multi-period corporate default prediction with stochastic covariates. Journal of Financial Economics, 83(3), 635-665.

[24] El Ghaoui, L., Oks M., \& Oustry, F. (2003) Worst-case value-at-risk and robust portfolio optimization: a conic programming approach. Operations research, 51(4), 543-556.

[25] Figlewski, S., Frydman, H., \& Liang, W. (2012). Modeling the effect of macroeconomic factors on corporate default and credit rating transitions. International Review of Economics \& Finance, 21(1), 87-105.

[26] Francis, W. B., \& Osborne, M. (2012). Capital requirements and bank behavior in the UK: Are there lessons for international capital standards? Journal of Banking \& Finance, 36(3), 803-816. 
[27] Frydman, H., \& Schuermann, T. (2008). Credit rating dynamics and Markov mixture models. Journal of Banking \& Finance, 32(6), 1062-1075.

[28] Gao, J., Zhou, K., Li, D., \& Cao, X. (2017). Dynamic mean-LPM and mean-CVaR portfolio optimization in continuous-time. SIAM Journal on Control and Optimization, 55(3), 1377-1397.

[29] Grant, M., Boyd, S., \& Ye Y. Y. (2008). CVX: Matlab software for disciplined convex programming. Web page and software, Available: http://cvxr.com/cvx/.

[30] Guidara, A., Soumaré, I., \& Tchana, F. T. (2013). Banks' capital buffer, risk and performance in the Canadian banking system: impact of business cycles and regulatory changes. Journal of Banking \& Finance, 37(9), 3373-3387.

[31] Gupton, G., Finger, C., \& Bhatia, M. (1997). CreditMetrics: the benchmark for understanding credit risk. Technical Document JP Morgan, New York.

[32] Heid, F. (2007). The cyclical effects of the Basel II capital requirements. Journal of Banking \& Finance, 31(12), 3885-3900.

[33] Hilscher, J., \& Wilson, M. (2017). Credit ratings and credit risk: is one measure enough? Management Science, 63(10), 3414-3437.

[34] Huang, X., \& Xiong, Q. (2015). Bank capital buffer decisions under macroeconomic fluctuations: evidence for the banking industry of China. International Review of Economics \& Finance, 36, 30-39.

[35] Hugonnier, J., \& Morellec, E. (2107). Bank capital, liquid reserves, and insolvency risk. Journal of Financial Economics, 125(2), 266-285.

[36] Ito, T., \& Sasaki, Y. N. (2002). Impacts of the Basle capital standard on Japanese banks' behavior. Journal of the Japanese and International Economies, 16(3), 372-397.

[37] Iscoe, I., Kreinin, A., Mausser, H., \& Romanko, O. (2012). Portfolio credit-risk optimization. Journal of Banking \& Finance, 36(6), 1604-1615.

[38] Jackson, P., Maude, D., \& Perraudin, W. (1998). Bank capital and value at risk. Bank of England working paper No. 79.

[39] James, C. M., \& Kizilaslan, A. (2014) Asset specificity, industry driven recovery risk and loan pricing. Journal of Financial and Quantitative Analysis, 49(03): 599-631.

[40] Kane, E., \& Malkiel, B. (1965). Bank portfolio allocation, deposit variability, and the availability doctrine. The Quarterly Journal of Economics, 79(1), 113-134.

[41] Kaplanski, G., \& Levy, H. (2015). Value-at-risk capital requirement regulation, risk taking and asset allocation: a mean-variance analysis. The European Journal of Finance, 21(3), 215-241.

[42] Khieu, H., Mullineaux, D., \& Yi, H. C. (2012). The determinants of bank loan recovery rates. Journal of Banking \& Finance 36(4), 923-933.

[43] Kolm, P., N., Tütüncü, R., \& Fabozzi, F. J. (2014). 60 Years of portfolio optimization: practical challenges and current trends. European Journal of Operational Research, 234(2), 356-371.

[44] Kusy, M., \& Ziemba, W. (1986). A bank asset and liability management model. Operations Research, 34(3), 356-376.

[45] Lu, M., Ran, L., \& Shen, Z.J.M. (2015). Reliable facility location design under uncertain correlated disruptions. Manufacturing\& Service Operations Management, 17(4), 445-455.

[46] Markowitz, H. (1952). Portfolio selection. The journal of finance, 7(1): 77-91.

[47] Mencía, J. Assessing the risk-return trade-off in loan portfolios. (2012). Journal of Banking \& Finance, 36(6): 1665-1677.

[48] Miller, B. L., \& Wagner, H. M. (1965). Chance constrained programming with joint constraints. Operations Research, 13(6), 930-945.

[49] Mukuddem-Petersen, J., \& Petersen, M.A. (2008). Optimizing asset and capital adequacy management in banking. Journal of Optimization Theory and Applications, 137(1), 205-230.

[50] Muller, G., E., \& Witbooi, P. J. (2014). An optimal portfolio and capital management strategy for Basel III compliant commercial banks. Journal of Applied Mathematics.

[51] Nemirovski, A., \& Shapiro A. (2006). Convex approximations of chance constrained programs. SIAM Journal on Optimization, 17(4), 969-996. 
[52] Oguzsoy, C., \& Gu, S. (1997). Bank asset and liability management under uncertainty. European Journal of Operational Research, 102(3), 575-600.

[53] Peura, S., \& Jokivuolle, E. (2004). Simulation based stress tests of banks' regulatory capital adequacy. Journal of Banking \& Finance, 28(8), 1801-1824.

[54] Pfeuffer, M., Reis, G., \& Smith, G.(2018). Capturing model risk and rating momentum in the estimation of probabilities of default and credit rating migrations. arXiv:1809.09889.

[55] Pu, X., \& Zhao, X. (2012). Correlation in credit risk changes. Journal of Banking \& Finance, 36(4), 1093-1106.

[56] Rockafellar, R.T. (1970). Convex Analysis, NJ, Princeton, Princeton University Press.

[57] Rockafellar, R.T., \& Uryasev, S. (2000). Optimization of conditional Value-at-Risk. The Journal of Risk, 2(3), 21-41.

[58] Sariannidis, N., Papadakis, S., Garefalakis, A., Lemonakis, C., \& Kyriaki-Argyro, T. (2019). Default avoidance on credit card portfolios using accounting, demographical and exploratory factors: decision making based on machine learning (ML) techniques. Annals of operational research, 1-25.

[59] Saunders, D., Xiouros, C., \& Zenios S. A. (2007). Credit risk optimization using factor models. Annals of Operations Research, 152(1): 49-77.

[60] Shapiro, A. (2001). On duality theory of conic linear problems. Nonconvex Optimization \& Its Applications 57, 135-165.

[61] Shim, J. (2013). Bank capital buffer and portfolio risk: the influence of business cycle and revenue diversification. Journal of Banking \& Finance, 37(3), 761-772.

[62] Singh, A., \& Dharmaraja, S. (2017). A portfolio optimisation model for credit risky bonds with Markov model credit rating dynamics. International Journal of Financial Markets and Derivatives, 6(2), 102-119.

[63] Sirignano, J.A., Tsoukalas, G., \& Giesecke, K. (2016). Large-scale loan portfolio selection. Operations Research, 64(6), 1239-1255.

[64] Sirignano J.A., \& Giesecke, K. (2018). Risk analysis for large pools of loans. Management Science, 65(1), 107-121.

[65] Sturm, J. F. (1999). Using SeDuMi 1.02, a MATLAB toolbox for optimization over symmetric cones. Optimization methods and software, 11(1-4): 625-653.

[66] Sun, Y., Loxton, R., \& Teo, KL. (2017). Chance-constrained optimization for pension fund portfolios in the presence of default risk. European Journal of Operational Research, 256(1), 205-214.

[67] Tang, D., Y., \& Yan, H. (2010). Market conditions, default risk and credit spreads. Journal of Banking \& Finance, 34(4), 743-753.

[68] Thomas, L. C. (2000) A survey of credit and behavioural scoring: forecasting financial risk of lending to consumers. International journal of forecasting, 16(2): 149-172.

[69] Toh, K. C., Todd, M. J., \& TütüncüR. H. (2012). On the implementation and usage of SDPT3-a Matlab software package for semidefinite-quadratic-linear programming, version 4.0, MA, Boston, Springer, 715-754.

[70] Westgaard, S., \& Van der Wijst N.V. (2001). Default probabilities in a corporate bank portfolio: A logistic model approach. European Journal of Operational Research, 135(2):338-349.

[71] Yao, H., Li Y., \& Benson, K. (2015). A smooth non-parametric estimation framework for safety-first portfolio optimization[J]. Quantitative Finance, 15(11): 1865-1884.

[72] Zymler, S., Kuhn, D., \& Rustem, B. (2013A). Worst-case value at risk of nonlinear portfolios. Management Science, 59(1), $172-188$.

[73] Zymler, S., Kuhn, D., \& Rustem, B. (2013B). Distributionally robust joint chance constraints with second-order moment information. Mathematical Programming, 137(1-2), 167-198. 
Table1 One-year corporate marginal transition rates for U.S. in 2007 (\%)

\begin{tabular}{ccccccccc}
\hline \multirow{2}{*}{ Initial Rating } & \multicolumn{7}{c}{ Ratings at the end of one year } \\
\cline { 2 - 8 } & $A A A$ & $A A$ & $A$ & $B B B$ & $B B$ & $B$ & $C C C$ & $D$ \\
\hline$A A A$ & 98.30 & 1.70 & 0 & 0 & 0 & 0 & 0 & 0 \\
$A A$ & 1.03 & 95.36 & 3.61 & 0 & 0 & 0 & 0 & 0 \\
$A$ & 0 & 3.61 & 92.25 & 3.25 & 0 & 0.72 & 0.18 & 0 \\
$B B B$ & 0 & 0.43 & 3.14 & 90.73 & 4.22 & 1.28 & 0 & 0 \\
$B B$ & 0 & 0 & 0 & 4.86 & 84.64 & 9.93 & 0.19 & 0.38 \\
$B$ & 0 & 0 & 0 & 0 & 6.75 & 89.89 & 3.24 & 0.13 \\
$C C C$ & 0 & 0 & 0 & 0 & 0 & 22.58 & 58.06 & 19.35 \\
\hline
\end{tabular}

Source: Standard \& Poor's report in analysis of transition rates (2007).

Table 2 One-year corporate marginal transition rates for U.S. in 2013 (\%)

\begin{tabular}{|c|c|c|c|c|c|c|c|c|}
\hline \multirow{2}{*}{ Initial Rating } & \multicolumn{8}{|c|}{ Ratings at the end of one year } \\
\hline & $A A A$ & $A A$ & $A$ & $B B B$ & $B B$ & $B$ & $C C C$ & $D$ \\
\hline$A A A$ & 1 & 0 & 0 & 0 & 0 & 0 & 0 & 0 \\
\hline$A A$ & 0 & 98.53 & 1.47 & 0 & 0 & 0 & 0 & 0 \\
\hline$A$ & 0 & 1.60 & 95.82 & 2.39 & 0.19 & 0 & 0 & 0 \\
\hline$B B B$ & 0 & 0 & 5.06 & 93.72 & 1.09 & 0.13 & 0 & 0 \\
\hline$B B$ & 0 & 0 & 0 & 6.72 & 90.14 & 3.14 & 0 & 0 \\
\hline$B$ & 0 & 0 & 0 & 0.33 & 6.05 & 88.90 & 3.73 & 0.99 \\
\hline$C C C$ & 0 & 0 & 0 & 0 & 0 & 14.67 & 52.00 & 33.33 \\
\hline
\end{tabular}

Source: Standard \& Poor's report in analysis of transition rates (2013).

Table 3 Term structure of forward rate at every non-default grade

\begin{tabular}{ccccc}
\hline Credit rating & 1-year forward $f_{\mathrm{r}}^{1,2}$ & 2-year forward $f_{\mathrm{r}}^{1,3}$ & 3-year forward $f_{\mathrm{r}}^{1,4}$ & 4.73 \\
\hline$A A A$ & 3.60 & 4.17 & 4.78 & 5.12 \\
$A A$ & 3.65 & 4.22 & 4.93 & 5.17 \\
$A$ & 3.72 & 4.32 & 5.25 & 5.32 \\
$B B B$ & 4.10 & 4.67 & 6.78 & 7.63 \\
$B B$ & 5.55 & 6.02 & 8.03 & 8.52 \\
$B$ & 6.05 & 7.02 & 14.03 & 13.52 \\
\hline
\end{tabular}

Source: Risk-Metrics Group Technical Document (2007). 
Table 4 Assets portfolio structure of the hypothetical bank in 2007

\begin{tabular}{|c|c|c|c|c|c|c|}
\hline Asset & $\begin{array}{c}\text { Collateral } \\
\text { type }\end{array}$ & $\begin{array}{c}\text { Recovery } \\
\text { rate }\end{array}$ & $\begin{array}{c}\text { Interest } \\
\text { rate }\end{array}$ & $\begin{array}{c}\text { Risk } \\
\text { weight }\end{array}$ & $\begin{array}{c}\text { Expected } \\
\text { value }\end{array}$ & $\begin{array}{l}\text { Standard } \\
\text { deviation }\end{array}$ \\
\hline \multicolumn{7}{|l|}{ Panel A Loan } \\
\hline (1) 5-year project financing (AA) & $\begin{array}{c}\text { Personal } \\
\text { guarantees }\end{array}$ & $60 \%$ & $7.39 \%$ & $100 \%$ & 1.1540 & 0.0055 \\
\hline (2) 5-year industrial loan (A) & $\begin{array}{l}\text { Accounts } \\
\text { receivable }\end{array}$ & $70 \%$ & $7.41 \%$ & $100 \%$ & 1.1467 & 0.0264 \\
\hline (3) 5-year land development loan (BBB) & Real property & $60 \%$ & $7.89 \%$ & $100 \%$ & 1.1517 & 0.0308 \\
\hline (4) 5-year business plant loan (BB) & Real property & $60 \%$ & $7.99 \%$ & $100 \%$ & 1.0950 & 0.0678 \\
\hline (5) 3-year loan for acquisition of other & Personal & $80 \%$ & $7.37 \%$ & $100 \%$ & 1.1332 & 0.0015 \\
\hline firms (AA) & property & & & & & \\
\hline (6) 3-year loan for purchasing of fixed & - & $60 \%$ & $7.39 \%$ & $100 \%$ & 1.1304 & 0.0180 \\
\hline \multicolumn{7}{|l|}{ assets (A) } \\
\hline (7) 3-year retailer financing (BBB) & Inventories & $85 \%$ & $7.88 \%$ & $75 \%$ & 1.1369 & 0.0104 \\
\hline (8) 3-year commercial loan (BB) & A-Rated ABS & $80 \%$ & $7.90 \%$ & $100 \%$ & 1.1089 & 0.0329 \\
\hline (9) 2-year retailer financing (AAA) & - & $60 \%$ & $7.32 \%$ & $75 \%$ & 1.1091 & $6.46 \mathrm{e}-05$ \\
\hline (10) 2-year corporate loan (A) & $\begin{array}{l}\text { Personal } \\
\text { property }\end{array}$ & $80 \%$ & $7.37 \%$ & $100 \%$ & 1.1084 & 0.0077 \\
\hline (11) 2-year personal loan (BBB) & $\begin{array}{c}\text { Personal } \\
\text { guarantees }\end{array}$ & $80 \%$ & $7.78 \%$ & $100 \%$ & 1.1124 & 0.0053 \\
\hline (12) 2-year retailer financing (BB) & $\begin{array}{c}\text { Government } \\
\text { bond }\end{array}$ & $80 \%$ & $7.88 \%$ & $75 \%$ & 1.0988 & 0.0253 \\
\hline \multicolumn{7}{|l|}{ Panel B Risk-free asset } \\
\hline (13) 1-year treasury bill (AAA) & - & $100 \%$ & $3.50 \%$ & 0 & 1.0350 & 0 \\
\hline
\end{tabular}

Note: Symbol "-" denotes no any collateral for the corresponding assets. 
Table 5 Assets portfolio structure of the hypothetical bank in 2013

\begin{tabular}{|c|c|c|c|c|c|c|}
\hline Asset & $\begin{array}{c}\text { Collateral } \\
\text { Type }\end{array}$ & $\begin{array}{c}\text { Recovery } \\
\text { rate }\end{array}$ & $\begin{array}{c}\text { Interest } \\
\text { rate }\end{array}$ & $\begin{array}{c}\text { Risk } \\
\text { weight }\end{array}$ & $\begin{array}{c}\text { Expected } \\
\text { Value }\end{array}$ & $\begin{array}{l}\text { Standard } \\
\text { deviation }\end{array}$ \\
\hline \multicolumn{7}{|l|}{ Panel A: Loan } \\
\hline (1) 5-year project financing & Personal & $65 \%$ & $4.39 \%$ & $100 \%$ & 1.0172 & $9.11 \mathrm{e}-04$ \\
\hline (AA) & guarantees & & & & & \\
\hline (2) 5-year industrial loan (A) & Accounts & $75 \%$ & $4.48 \%$ & $100 \%$ & 1.0164 & 0.0047 \\
\hline & receivable & & & & & \\
\hline (3) 5-year land development loan & Real property & $65 \%$ & $4.92 \%$ & $100 \%$ & 1.0257 & 0.0112 \\
\hline \multicolumn{7}{|l|}{ (BBB) } \\
\hline (4) 5 -year business plant loan (BB) & Real property & $65 \%$ & $5.04 \%$ & $100 \%$ & 0.9817 & 0.0314 \\
\hline (5) 3-year loan for acquisition of other & Personal & $85 \%$ & $4.36 \%$ & $100 \%$ & 1.0464 & $2.88 \mathrm{e}-04$ \\
\hline firms (AA) & property & & & & & \\
\hline (6) 3-year loan for purchasing of fixed & - & $65 \%$ & $4.40 \%$ & $100 \%$ & 1.0455 & 0.0019 \\
\hline \multicolumn{7}{|l|}{ assets (A) } \\
\hline (7) 3-year retailer financing (BBB) & Inventories & $90 \%$ & $4.90 \%$ & $75 \%$ & 1.0536 & 0.0040 \\
\hline (8) 3-year commercial loan (BB) & A-RatedABS & $85 \%$ & $4.92 \%$ & $100 \%$ & 1.0304 & 0.0108 \\
\hline (9) 2-year retailer financing (AAA) & - & $65 \%$ & $4.01 \%$ & $75 \%$ & 1.0441 & 0 \\
\hline (10) 2-year corporate loan (A) & Personal & $85 \%$ & $4.37 \%$ & $100 \%$ & 1.0499 & $9.56 \mathrm{e}-04$ \\
\hline & property & & & & & \\
\hline (11) 2-year personal loan (BBB) & Personal & $85 \%$ & $4.88 \%$ & $100 \%$ & 1.0563 & 0.0019 \\
\hline & guarantees & & & & & \\
\hline (12) 2-year retailer financing (BB) & Government & $85 \%$ & $4.90 \%$ & $75 \%$ & 1.0436 & 0.0050 \\
\hline & bond & & & & & \\
\hline \multicolumn{7}{|l|}{ Panel B: Risk-free asset } \\
\hline (13) 1-year treasury bill(AAA) & - & $100 \%$ & $0.13 \%$ & 0 & 1.0013 & 0 \\
\hline
\end{tabular}

Note: Symbol “-” denotes no any collateral for the corresponding assets. 
Table 6 Recovery rate setting rules

\begin{tabular}{llcc}
\hline Maturity & \multicolumn{1}{c}{ Collateral style } & $\begin{array}{c}\text { Recovery rate } \\
\text { in 2007 }\end{array}$ & $\begin{array}{c}\text { Recovery rate } \\
\text { in 2013 }\end{array}$ \\
\hline$\geq 5$ year & (1) inventories or accounts receivable & $70 \%$ & $75 \%$ \\
& (2) other collateral except for above two & $60 \%$ & $65 \%$ \\
& (3) non-collateral & $55 \%$ & $60 \%$ \\
\hline $1 \sim 3$ year & (1) inventories or accounts receivable & $85 \%$ & $90 \%$ \\
& (2) other collateral except for above two & $80 \%$ & $85 \%$ \\
\hline$\leq 1$ year & (3) non-collateral & $60 \%$ & $65 \%$ \\
\hline & (1) inventories or accounts receivable & $95 \%$ & $100 \%$ \\
& (2) other collateral except for above two & $90 \%$ & $95 \%$ \\
\hline
\end{tabular}

Table 7 Optimal assets allocation for the hypothetical bank in 2007 and 2013

\begin{tabular}{lllllllllllllll}
\hline Year & $x_{1}$ & $x_{2}$ & $x_{3}$ & $x_{4}$ & $x_{5}$ & $x_{6}$ & $x_{7}$ & $x_{8}$ & $x_{9}$ & $x_{10}$ & $x_{11}$ & $x_{12}$ & $x_{13}$ & $R o R$ \\
\hline 2007 & 0 & 0 & 0.0979 & 0 & 0.0521 & 0 & 0.2 & 0 & 0 & 0 & 0.2 & 0.2 & 0.25 & $6.7394 \%$ \\
2013 & 0 & 0 & 0 & 0.0746 & 0 & 0 & 0.2 & 0.0754 & 0 & 0 & 0.2 & 0.2 & 0.25 & $3.7155 \%$ \\
& $(-)$ & $(-)$ & $(\downarrow)$ & $(\uparrow)$ & $(\downarrow)$ & $(-)$ & $(-)$ & $(\uparrow)$ & $(-)$ & $(-)$ & $(-)$ & $(-)$ & $(-)$ & \\
\hline
\end{tabular}

Note: The symbols in the brackets denote the changing tendency of proportion allocated to 12 loans compared with that for 2007 , and “-", " $\downarrow$ " and " $\uparrow "$ denote unchanged, decreasing and increasing respectively; "RoR" stands for the maximum portfolio expected rate of return and the same below. 


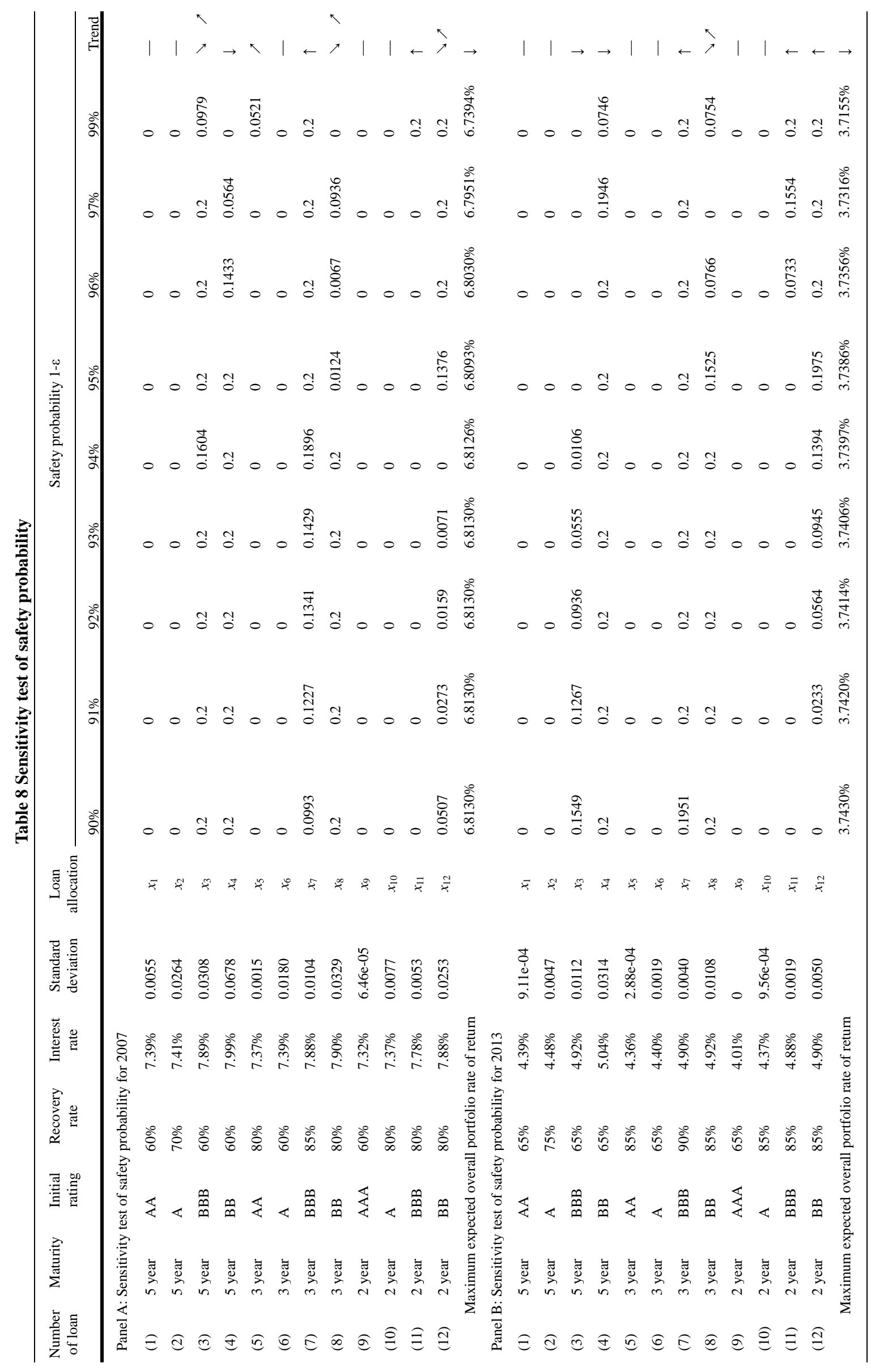




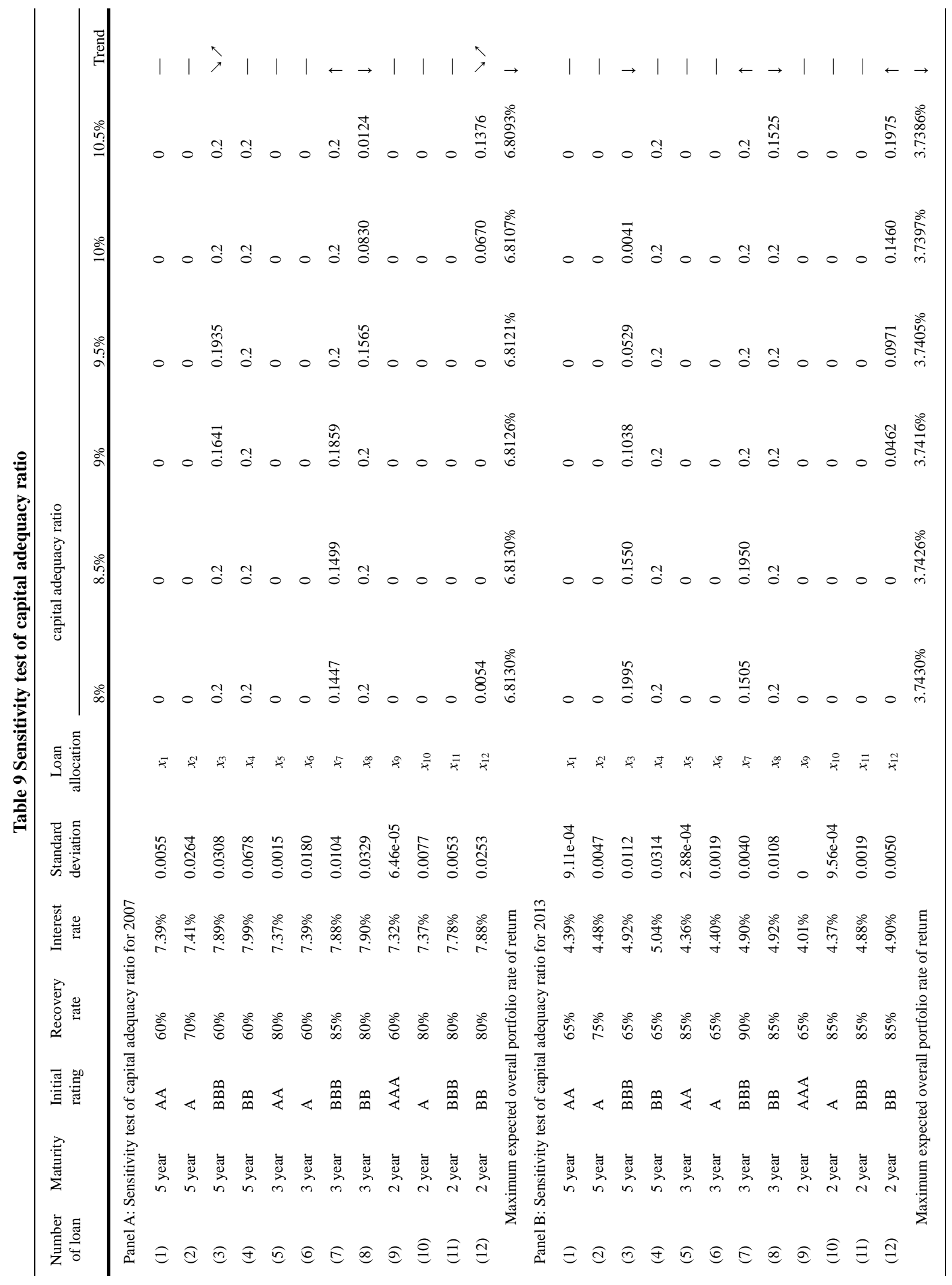


Table 10 Sensitivity test of recovery rate with safety probability of $95 \%$ in 2007

\begin{tabular}{|c|c|c|c|c|c|c|c|c|c|c|c|}
\hline \multirow{2}{*}{$\begin{array}{l}\text { Loan } 4 \\
\text { 5-year BB }\end{array}$} & \multicolumn{5}{|c|}{ Recovery rate } & \multirow{2}{*}{$\begin{array}{l}\text { Loan } 3 \\
\text { 5-year BBB }\end{array}$} & \multicolumn{5}{|c|}{ Recovery rate } \\
\hline & $50 \%$ & $70 \%$ & $80 \%$ & $85 \%$ & Trend & & $50 \%$ & $70 \%$ & $80 \%$ & $85 \%$ & Trend \\
\hline Mean & 1.0931 & 1.0968 & 1.0987 & 1.0996 & $\uparrow$ & Mean & 1.1515 & 1.1519 & 1.1521 & 1.1522 & $\uparrow$ \\
\hline Std. & 0.0796 & 0.0564 & 0.0445 & 0.0404 & $\downarrow$ & Std. & 0.0337 & 0.0281 & 0.0258 & 0.0248 & $\downarrow$ \\
\hline$x_{1}(7.39 \%)$ & 0 & 0 & 0 & 0 & - & $x_{1}(7.39 \%)$ & 0 & 0 & 0 & 0 & - \\
\hline$x_{2}(7.41 \%)$ & 0 & 0 & 0 & 0 & - & $x_{2}(7.41 \%)$ & 0 & 0 & 0 & 0 & - \\
\hline$x_{3}(7.89 \%)$ & 0.2 & 0.1550 & 0.2 & 0.2 & $\searrow \nearrow$ & $x_{3}(7.89 \%)$ & 0.2 & 0.2 & 0.2 & 0.2 & - \\
\hline$x_{4}(7.99 \%)$ & 0.1631 & 0.2 & 0.2 & 0.2 & $\uparrow$ & $x_{4}(7.99 \%)$ & 0.1912 & 0.2 & 0.2 & 0.2 & $\uparrow$ \\
\hline$x_{5}(7.37 \%)$ & 0 & 0 & 0 & 0 & - & $x_{5}(7.37 \%)$ & 0 & 0 & 0 & 0 & - \\
\hline$x_{6}(7.39 \%)$ & 0 & 0 & 0 & 0 & - & $x_{6}(7.39 \%)$ & 0 & 0 & 0 & 0 & - \\
\hline$x_{7}(7.88 \%)$ & 0.2 & 0.1950 & 0.1424 & 0.1379 & $\downarrow$ & $x_{7}(7.88 \%)$ & 0.2 & 0.2 & 0.2 & 0.2 & - \\
\hline$x_{8}(7.90 \%)$ & 0 & 0.2 & 0.2 & 0.2 & $\uparrow$ & $x_{8}(7.90 \%)$ & 0 & 0.0584 & 0.0977 & 0.1149 & $\uparrow$ \\
\hline$x_{9}(7.32 \%)$ & 0 & 0 & 0 & 0 & - & $x_{9}(7.32 \%)$ & 0 & 0 & 0 & 0 & - \\
\hline$x_{10}(7.37 \%)$ & 0 & 0 & 0 & 0 & - & $x_{10}(7.37 \%)$ & 0 & 0 & 0 & 0 & - \\
\hline$x_{11}(7.78 \%)$ & 0 & 0 & 0 & 0 & - & $x_{11}(7.78 \%)$ & 0 & 0 & 0 & 0 & - \\
\hline$x_{12}(7.88 \%)$ & 0.1869 & 0 & 0.0076 & 0.0121 & $\searrow \nearrow$ & $x_{12}(7.88 \%)$ & 0.1588 & 0.0916 & 0.0523 & 0.0351 & $\downarrow$ \\
\hline$R o R$ & $6.8049 \%$ & $6.8126 \%$ & $6.8130 \%$ & $6.8130 \%$ & $\uparrow$ & $R o R$ & $6.8080 \%$ & $6.8102 \%$ & $6.8110 \%$ & $6.8113 \%$ & $\uparrow$ \\
\hline Loan 11 & \multicolumn{5}{|c|}{ Recovery rate } & Loan 5 & \multicolumn{5}{|c|}{ Recovery rate } \\
\hline 2-year BBB & $60 \%$ & $70 \%$ & $80 \%$ & $95 \%$ & Trend & 3-year AA & $60 \%$ & $70 \%$ & $80 \%$ & $95 \%$ & Trend \\
\hline Mean & 1.1123 & 1.1123 & 1.1124 & 1.1124 & $\uparrow$ & Mean & 1.1332 & 1.1332 & 1.1332 & 1.1332 & - \\
\hline Std. & 0.0074 & 0.0063 & 0.0053 & 0.0041 & $\downarrow$ & Std. & 0.0020 & 0.0017 & 0.0015 & 0.0011 & $\downarrow$ \\
\hline$x_{1}(7.39 \%)$ & 0 & 0 & 0 & 0 & - & $x_{1}(7.39 \%)$ & 0 & 0 & 0 & 0 & - \\
\hline$x_{2}(7.41 \%)$ & 0 & 0 & 0 & 0 & - & $x_{2}(7.41 \%)$ & 0 & 0 & 0 & 0 & - \\
\hline$x_{3}(7.89 \%)$ & 0.2 & 0.2 & 0.2 & 0.2 & - & $x_{3}(7.89 \%)$ & 0.2 & 0.2 & 0.2 & 0.2 & - \\
\hline$x_{4}(7.99 \%)$ & 0.2 & 0.2 & 0.2 & 0.2 & - & $x_{4}(7.99 \%)$ & 0.2 & 0.2 & 0.2 & 0.2 & - \\
\hline$x_{5}(7.37 \%)$ & 0 & 0 & 0 & 0 & - & $x_{5}(7.37 \%)$ & 0 & 0 & 0 & 0 & - \\
\hline$x_{6}(7.39 \%)$ & 0 & 0 & 0 & 0 & - & $x_{6}(7.39 \%)$ & 0 & 0 & 0 & 0 & - \\
\hline$x_{7}(7.88 \%)$ & 0.2 & 0.2 & 0.2 & 0.2 & - & $x_{7}(7.88 \%)$ & 0.2 & 0.2 & 0.2 & 0.2 & - \\
\hline$x_{8}(7.90 \%)$ & 0.0124 & 0.0124 & 0.0124 & 0.0124 & - & $x_{8}(7.90 \%)$ & 0.0124 & 0.10124 & 0.0124 & 0.0124 & - \\
\hline$x_{9}(7.32 \%)$ & 0 & 0 & 0 & 0 & - & $x_{9}(7.32 \%)$ & 0 & 0 & 0 & 0 & - \\
\hline$x_{10}(7.37 \%)$ & 0 & 0 & 0 & 0 & - & $x_{10}(7.37 \%)$ & 0 & 0 & 0 & 0 & - \\
\hline$x_{11}(7.78 \%)$ & 0 & 0 & 0 & 0 & - & $x_{11}(7.78 \%)$ & 0 & 0 & 0 & 0 & - \\
\hline$x_{12}(7.88 \%)$ & 0.1376 & 0.1376 & 0.1376 & 0.1376 & - & $x_{12}(7.88 \%)$ & 0.1376 & 0.1376 & 0.1376 & 0.1376 & - \\
\hline RoR & $6.8093 \%$ & $6.8093 \%$ & $6.8093 \%$ & $6.8093 \%$ & - & $R o R$ & $6.8093 \%$ & $6.8093 \%$ & $6.8093 \%$ & $6.8093 \%$ & - \\
\hline
\end{tabular}


Table 11 Sensitivity test of recovery rate with safety probability of $99 \%$ in 2007

\begin{tabular}{|c|c|c|c|c|c|c|c|c|c|c|c|}
\hline \multirow{2}{*}{$\begin{array}{l}\text { Loan } 4 \\
\text { 5-year } B B\end{array}$} & \multicolumn{5}{|c|}{ Recovery rate } & \multirow{2}{*}{$\begin{array}{l}\text { Loan } 3 \\
\text { 5-year } B B B\end{array}$} & \multicolumn{5}{|c|}{ Recovery rate } \\
\hline & $50 \%$ & $70 \%$ & $80 \%$ & $85 \%$ & Trend & & $50 \%$ & $70 \%$ & $80 \%$ & $85 \%$ & Trend \\
\hline Mean & 1.0931 & 1.0968 & 1.0987 & 1.0996 & $\uparrow$ & Mean & 1.1515 & 1.1519 & 1.1521 & 1.1522 & $\uparrow$ \\
\hline Std. & 0.0796 & 0.0564 & 0.0455 & 0.0404 & $\downarrow$ & Std. & 0.0337 & 0.0281 & 0.0258 & 0.0248 & $\downarrow$ \\
\hline$x_{1}(7.39 \%)$ & 0 & 0 & 0 & 0 & - & $x_{1}(7.39 \%)$ & 0 & 0 & 0 & 0 & - \\
\hline$x_{2}(7.41 \%)$ & 0 & 0 & 0 & 0 & - & $x_{2}(7.41 \%)$ & 0 & 0 & 0 & 0 & - \\
\hline$x_{3}(7.89 \%)$ & 0.0976 & 0.0978 & 0.0978 & 0.0978 & $\uparrow$ & $x_{3}(7.89 \%)$ & 0.0883 & 0.2 & 0.2 & 0.2 & $\uparrow$ \\
\hline$x_{4}(7.99 \%)$ & 0 & 0 & 0 & 0 & - & $x_{4}(7.99 \%)$ & 0 & 0 & 0 & 0 & - \\
\hline$x_{5}(7.37 \%)$ & 0.0524 & 0.0522 & 0.0522 & 0.0522 & $\downarrow$ & $x_{5}(7.37 \%)$ & 0.0617 & 0.0412 & 0.0223 & 0.0156 & $\downarrow$ \\
\hline$x_{6}(7.39 \%)$ & 0 & 0 & 0 & 0 & - & $x_{6}(7.39 \%)$ & 0 & 0 & 0 & 0 & - \\
\hline$x_{7}(7.88 \%)$ & 0.2 & 0.2 & 0.2 & 0.2 & - & $x_{7}(7.88 \%)$ & 0.2 & 0.2 & 0.2 & 0.2 & - \\
\hline$x_{8}(7.90 \%)$ & 0 & 0 & 0 & 0 & - & $x_{8}(7.90 \%)$ & 0 & 0 & 0 & 0 & - \\
\hline$x_{9}(7.32 \%)$ & 0 & 0 & 0 & 0 & - & $x_{9}(7.32 \%)$ & 0 & 0 & 0.0001 & 0 & - \\
\hline$x_{10}(7.37 \%)$ & 0 & 0 & 0 & 0 & - & $x_{10}(7.37 \%)$ & 0 & 0 & 0 & 0 & - \\
\hline$x_{11}(7.78 \%)$ & 0.2 & 0.2 & 0.2 & 0.2 & - & $x_{11}(7.78 \%)$ & 0.2 & 0.2 & 0.2 & 0.2 & - \\
\hline$x_{12}(7.88 \%)$ & 0.2 & 0.2 & 0.2 & 0.2 & - & $x_{12}(7.88 \%)$ & 0.2 & 0.1088 & 0.1275 & 0.1344 & $\searrow \nearrow$ \\
\hline RoR & $6.7393 \%$ & $6.7394 \%$ & $6.7394 \%$ & $6.7394 \%$ & $\uparrow$ & $R o R$ & $6.7344 \%$ & $6.7460 \%$ & $6.7555 \%$ & $6.7590 \%$ & $\uparrow$ \\
\hline Loan 11 & \multicolumn{5}{|c|}{ Recovery rate } & Loan 5 & \multicolumn{5}{|c|}{ Recovery rate } \\
\hline 2-year $B B B$ & $60 \%$ & $70 \%$ & $80 \%$ & $95 \%$ & Trend & 3-year $A A$ & $60 \%$ & $70 \%$ & $80 \%$ & $95 \%$ & Trend \\
\hline Mean & 1.1123 & 1.1123 & 1.1124 & 1.1125 & $\uparrow$ & Mean & 1.1332 & 1.1332 & 1.1332 & 1.1332 & - \\
\hline Std. & 0.0074 & 0.0063 & 0.0053 & 0.0041 & $\downarrow$ & Std. & 0.0020 & 0.0017 & 0.0015 & 0.0011 & $\downarrow$ \\
\hline$x_{1}(7.39 \%)$ & 0 & 0 & 0 & 0 & - & $x_{1}(7.39 \%)$ & 0 & 0 & 0 & 0 & - \\
\hline$x_{2}(7.41 \%)$ & 0 & 0 & 0 & 0 & - & $x_{2}(7.41 \%)$ & 0 & 0 & 0 & 0 & $\uparrow$ \\
\hline$x_{3}(7.89 \%)$ & 0.0814 & 0.0905 & 0.0979 & 0.1064 & $\uparrow$ & $x_{3}(7.89 \%)$ & 0.1016 & 0.0974 & 0.0979 & 0.0987 & $\searrow \nearrow$ \\
\hline$x_{4}(7.99 \%)$ & 0 & 0 & 0 & 0 & - & $x_{4}(7.99 \%)$ & 0 & 0 & 0 & 0 & - \\
\hline$x_{5}(7.37 \%)$ & 0.0686 & 0.0595 & 0.0521 & 0.0436 & $\downarrow$ & $x_{5}(7.37 \%)$ & 0.0023 & 0.0526 & 0.0521 & 0.0513 & $\nearrow \searrow$ \\
\hline$x_{6}(7.39 \%)$ & 0 & 0 & 0 & 0 & - & $x_{6}(7.39 \%)$ & 0 & 0 & 0 & 0 & - \\
\hline$x_{7}(7.88 \%)$ & 0.2 & 0.2 & 0.2 & 0.2 & - & $x_{7}(7.88 \%)$ & 0.2 & 0.2 & 0.2 & 0.2 & - \\
\hline$x_{8}(7.90 \%)$ & 0 & 0 & 0 & 0 & - & $x_{8}(7.90 \%)$ & 0 & 0 & 0 & 0 & - \\
\hline$x_{9}(7.32 \%)$ & 0 & 0 & 0 & 0 & - & $x_{9}(7.32 \%)$ & 0.0461 & 0 & 0 & 0 & $\downarrow$ \\
\hline$x_{10}(7.37 \%)$ & 0 & 0 & 0 & 0 & - & $x_{10}(7.37 \%)$ & 0 & 0 & 0 & 0 & - \\
\hline$x_{11}(7.78 \%)$ & 0.2 & 0.2 & 0.2 & 0.2 & - & $x_{11}(7.78 \%)$ & 0.2 & 0.2 & 0.2 & 0.2 & - \\
\hline$x_{12}(7.88 \%)$ & 0.2 & 0.2 & 0.2 & 0.2 & - & $x_{12}(7.88 \%)$ & 0.2 & 0.2 & 0.2 & 0.2 & - \\
\hline$R o R$ & $6.7308 \%$ & $6.7355 \%$ & $6.7394 \%$ & $6.7439 \%$ & $\uparrow$ & $R o R$ & $6.7390 \%$ & $6.7392 \%$ & $6.7394 \%$ & $6.7398 \%$ & $\uparrow$ \\
\hline
\end{tabular}


Table 12 Sensitivity test of changing recovery rates for multiple loans with safety probability of $95 \%$ in 2007

\begin{tabular}{|c|c|c|c|c|c|c|c|}
\hline & Interest rate & Recovery rate & Expected value & Allocation & Recovery rate & Expected value & Allocation \\
\hline$x_{1}$ & $7.39 \%$ & $60 \%$ & 1.154 & 0 & $60 \%$ & 1.154 & 0 \\
\hline$x_{2}$ & $7.41 \%$ & $70 \%$ & 1.1467 & 0 & $70 \%$ & 1.1467 & 0 \\
\hline$x_{3}$ & $7.89 \%$ & $60 \%$ & 1.1517 & 0.2 & $50 \%$ & 1.1515 & 0.2 \\
\hline$x_{4}$ & $7.99 \%$ & $60 \%$ & 1.095 & 0.2 & $50 \%$ & 1.0931 & 0.0978 \\
\hline$x_{5}$ & $7.37 \%$ & $80 \%$ & 1.1332 & 0 & $80 \%$ & 1.1332 & 0 \\
\hline$x_{6}$ & $7.39 \%$ & $60 \%$ & 1.1304 & 0 & $60 \%$ & 1.1304 & 0 \\
\hline$x_{7}$ & $7.88 \%$ & $85 \%$ & 1.1369 & 0.2 & $60 \%$ & 1.1368 & 0.2 \\
\hline$x_{8}$ & $7.90 \%$ & $80 \%$ & 1.1089 & 0.0124 & $60 \%$ & 1.1067 & 0 \\
\hline$x_{9}$ & $7.32 \%$ & $60 \%$ & 1.1091 & 0 & $60 \%$ & 1.1091 & 0 \\
\hline$x_{10}$ & $7.37 \%$ & $80 \%$ & 1.1084 & 0 & $80 \%$ & 1.1084 & 0 \\
\hline$x_{11}$ & $7.78 \%$ & $80 \%$ & 1.1124 & 0 & $80 \%$ & 1.1124 & 0.0522 \\
\hline$x_{12}$ & $7.88 \%$ & $80 \%$ & 1.0988 & 0.1376 & $60 \%$ & 1.0973 & 0.2 \\
\hline RoR & & & & $6.8093 \%$ & & & $6.7925 \%$ \\
\hline
\end{tabular}

Table 13 Sensitivity test of changing recovery rates for multiple loans with safety probability of $99 \%$ in 2007

\begin{tabular}{lcccc|ccc}
\hline & Interest rate & Recovery rate & Expected value & Allocation & Recovery rate & Expected value & Allocation \\
\hline$x_{1}$ & $7.39 \%$ & $60 \%$ & 1.154 & 0 & $60 \%$ & 1.154 & 0 \\
$x_{2}$ & $7.41 \%$ & $70 \%$ & 1.1467 & 0 & $70 \%$ & 1.1467 & 0 \\
$x_{3}$ & $7.89 \%$ & $60 \%$ & 1.1517 & 0.0979 & $60 \%$ & 1.1517 & 0.2 \\
$x_{4}$ & $7.99 \%$ & $60 \%$ & 1.095 & 0 & $60 \%$ & 1.095 & 0 \\
$x_{5}$ & $7.37 \%$ & $80 \%$ & 1.1332 & 0.0521 & $95 \%$ & 1.1332 & 0 \\
$x_{6}$ & $7.39 \%$ & $60 \%$ & 1.1304 & 0 & $60 \%$ & 1.1304 & 0 \\
$x_{7}$ & $7.88 \%$ & $85 \%$ & 1.1369 & 0.2 & $85 \%$ & 1.1369 & 0.2 \\
$x_{8}$ & $7.90 \%$ & $80 \%$ & 1.1089 & 0 & $80 \%$ & 1.1089 & 0.006 \\
$x_{9}$ & $7.32 \%$ & $60 \%$ & 1.1091 & 0 & $60 \%$ & 1.1091 & 0 \\
$x_{10}$ & $7.37 \%$ & $80 \%$ & 1.1084 & 0 & $80 \%$ & 1.1084 & 0 \\
$x_{11}$ & $7.78 \%$ & $80 \%$ & 1.1124 & 0.2 & $95 \%$ & 1.1124 & 0.144 \\
$x_{12}$ & $7.88 \%$ & $80 \%$ & 1.0988 & 0.2 & $95 \%$ & & 1.0999 \\
\hline RoR & & & $6.7394 \%$ & & & 0.2 \\
\hline
\end{tabular}

Table 14 Optimal asset allocation and performance of CVaR model, $\beta=95 \%$

\begin{tabular}{|c|c|c|c|c|c|c|c|c|c|c|c|c|c|c|}
\hline Smpls (\#) & $x_{1}$ & $x_{2}$ & $x_{3}$ & $x_{4}$ & $x_{5}$ & $x_{6}$ & $x_{7}$ & $x_{8}$ & $x_{9}$ & $x_{10}$ & $x_{11}$ & $x_{12}$ & CVaR & elapsed time \\
\hline 1,000 & 0.0832 & 0.0011 & 0.0102 & 0.0069 & 0.2 & 0.0324 & 0.1708 & 0.0142 & 0 & 0.0132 & 0.2 & 0.0180 & 0.0076 & $2.4 \mathrm{sec}$ \\
\hline 5,000 & 0.1221 & 0 & 0.0106 & 0.0055 & 0.2 & 0.0091 & 0.1708 & 0.0150 & 0 & 0 & 0.2 & 0.0169 & 0.0076 & $58.8 \mathrm{sec}$ \\
\hline 10,000 & 0.0124 & 0 & 0.0102 & 0.0063 & 0.2 & 0.0091 & 0.1800 & 0.0150 & 0.0521 & 0.0476 & 0.2 & 0.0173 & 0.0075 & $376 \mathrm{sec}$ \\
\hline 20,000 & 0.0671 & 0 & 0.0100 & 0.0053 & 0.2 & 0.0080 & 0.1800 & 0.0150 & 0.0342 & 0.0140 & 0.2 & 0.0164 & 0.0075 & $3117 \mathrm{sec}$ \\
\hline
\end{tabular}

Table 15 Optimal asset allocation and performance of CVaR model, $\beta=99 \%$

\begin{tabular}{|c|c|c|c|c|c|c|c|c|c|c|c|c|c|c|}
\hline Smpls (\#) & $x_{1}$ & $x_{2}$ & $x_{3}$ & $x_{4}$ & $x_{5}$ & $x_{6}$ & $x_{7}$ & $x_{8}$ & $x_{9}$ & $x_{10}$ & $x_{11}$ & $x_{12}$ & CVaR & elapsed time \\
\hline 1,000 & 0.2 & 0 & 0.0144 & 0.0107 & 0.0502 & 0.0365 & 0.1569 & 0.0126 & 0 & 0.0477 & 0.2 & 0.0210 & 0.0129 & $2.9 \mathrm{sec}$ \\
\hline 5,000 & 0.1 & 0 & 0.0145 & 0.0052 & 0.2 & 0.0134 & 0.1622 & 0.0188 & 0 & 0.0173 & 0.2 & 0.0186 & 0.0105 & $87.8 \mathrm{sec}$ \\
\hline 10,000 & 0.0130 & 0.0091 & 0.0151 & 0.0043 & 0.2 & 0 & 0.1637 & 0.0196 & 0.0362 & 0.0652 & 0.2 & 0.0238 & 0.0114 & $586 \mathrm{sec}$ \\
\hline 20,000 & 0.0180 & 0.0100 & 0.0150 & 0.0073 & 0.2 & 0.0064 & 0.1676 & 0.0174 & 0.0673 & 0.0200 & 0.2 & 0.0210 & 0.0132 & $3137 \mathrm{sec}$ \\
\hline
\end{tabular}


Table 16 Performance of CVaR model on CAR, $\beta=99 \%$ and $J=20,000$

\begin{tabular}{ccccccc}
\hline TL & Max & Mean & Min & $\leq 15 \%(\#)$ & $\leq 10.5 \%(\#)$ & $\leq 8.0 \%(\#)$ \\
\hline$T L_{1}(1,438,926)$ & $23.84 \%$ & $23.50 \%$ & $17.10 \%$ & 0 & 0 & 0 \\
$T L_{2}(1,463,570)$ & $21.80 \%$ & $21.45 \%$ & $14.85 \%$ & 5 & 0 & 0 \\
$T L_{3}(1,488,214)$ & $19.76 \%$ & $19.40 \%$ & $12.64 \%$ & 17 & 0 & 0 \\
$T L_{4}(1,512,858)$ & $17.72 \%$ & $17.36 \%$ & $9.42 \%$ & 20 & 3 & 0 \\
\hline
\end{tabular}

Table 17 Optimal asset allocation and performance of our model, $\beta=95 \%$ and target $\mathrm{CAR}=10.5 \%$

\begin{tabular}{ccccccccccccccc}
\hline TL & $x_{1}$ & $x_{2}$ & $x_{3}$ & $x_{4}$ & $x_{5}$ & $x_{6}$ & $x_{7}$ & $x_{8}$ & $x_{9}$ & $x_{10}$ & $x_{11}$ & $x_{12}$ & RoR & elapsed time \\
\hline$T L_{1}(1,438,926)$ & 0 & 0 & 0.2 & 0.2 & 0 & 0 & 0.2 & 0.0124 & 0 & 0 & 0 & 0.1376 & $6.8093 \%$ & $0.45 \mathrm{sec}$ \\
$T L_{2}(1,463,570)$ & 0 & 0 & 0.2 & 0.1134 & 0 & 0 & 0.2 & 0.0366 & 0 & 0 & 0 & 0.2 & $6.8002 \%$ & $0.47 \mathrm{sec}$ \\
$T L_{3}(1,488,214)$ & 0 & 0 & 0.2 & 0.0072 & 0 & 0 & 0.2 & 0.1428 & 0 & 0 & 0 & 0.2 & $6.7906 \%$ & $0.45 \mathrm{sec}$ \\
$T L_{4}(1,512,858)$ & 0 & 0 & 0.2 & 0 & 0 & 0 & 0.2 & 0.0173 & 0 & 0 & 0.1327 & 0.2 & $6.7741 \%$ & $0.48 \mathrm{sec}$ \\
\hline
\end{tabular}

Table 18 Optimal asset allocation and performance of our model, $\beta=99 \%$, target $\mathrm{CAR}=10.5 \%$

\begin{tabular}{|c|c|c|c|c|c|c|c|c|c|c|c|c|c|c|}
\hline $\mathrm{TL}$ & $x_{1}$ & $x_{2}$ & $x_{3}$ & $x_{4}$ & $x_{5}$ & $x_{6}$ & $x_{7}$ & $x_{8}$ & $x_{9}$ & $x_{10}$ & $x_{11}$ & $x_{12}$ & RoR & elapsed time \\
\hline$T L_{1}(1,438,926)$ & 0 & 0 & 0.0979 & 0 & 0.0521 & 0 & 0.2 & 0 & 0 & 0 & 0.2 & 0.2 & $6.7394 \%$ & $0.52 \mathrm{sec}$ \\
\hline$T L_{2}(1,463,570)$ & 0 & 0 & 0.0332 & 0 & 0.1168 & 0 & 0.2 & 0 & 0 & 0 & 0.2 & 0.2 & $6.7058 \%$ & $0.53 \mathrm{sec}$ \\
\hline$T L_{3}(1,488,214)$ & 0 & 0 & 0 & 0 & 0.1838 & 0 & 0.2 & 0 & 0.0010 & 0 & 0.2 & 0.1652 & $6.6707 \%$ & $0.59 \mathrm{sec}$ \\
\hline$T L_{4}(1,512,858)$ & 0 & 0 & 0 & 0 & 0.2 & 0 & 0.2 & 0 & 0.0515 & 0 & 0.2 & 0.0985 & $6.6342 \%$ & $0.52 \mathrm{sec}$ \\
\hline \multicolumn{15}{|c|}{ Table 19 Performance of our model on CAR, $\beta=99 \%$ and $J=20,000$} \\
\hline \multicolumn{2}{|l|}{$\mathrm{TL}$} & \multicolumn{2}{|r|}{ Max } & \multicolumn{3}{|c|}{ Mean } & \multicolumn{2}{|c|}{ Min } & \multicolumn{2}{|c|}{$\leq 15 \%(\#)$} & \multicolumn{3}{|c|}{$\leq 10.5 \%(\#)$} & $\leq 8.0 \%(\#)$ \\
\hline \multicolumn{2}{|l|}{$T L_{1}(1,438,926)$} & \multicolumn{2}{|r|}{$25.33 \%$} & \multicolumn{3}{|c|}{$24.53 \%$} & \multicolumn{2}{|c|}{$16.43 \%$} & \multicolumn{2}{|c|}{0} & \multicolumn{3}{|c|}{0} & 0 \\
\hline \multicolumn{2}{|l|}{$T L_{2}(1,463,570)$} & \multicolumn{2}{|r|}{$22.95 \%$} & \multicolumn{3}{|c|}{$22.25 \%$} & \multicolumn{2}{|c|}{$13.56 \%$} & \multicolumn{2}{|c|}{29} & \multicolumn{3}{|c|}{0} & 0 \\
\hline \multicolumn{2}{|l|}{$T L_{3}(1,488,214)$} & \multicolumn{2}{|r|}{$20.51 \%$} & \multicolumn{3}{|c|}{$19.89 \%$} & \multicolumn{2}{|c|}{$12.84 \%$} & \multicolumn{2}{|c|}{152} & \multicolumn{3}{|c|}{0} & 0 \\
\hline \multicolumn{2}{|l|}{$T L_{4}(1,512,858)$} & \multicolumn{2}{|r|}{$18.33 \%$} & \multicolumn{3}{|c|}{$17.77 \%$} & \multicolumn{2}{|c|}{$11.31 \%$} & \multicolumn{2}{|c|}{146} & \multicolumn{3}{|c|}{0} & 0 \\
\hline
\end{tabular}

Table 20 Optimal asset allocation of CVaR model, estimated by different MC simulations

\begin{tabular}{clllllllllllllll}
\hline Type of distribution & $x_{1}$ & $x_{2}$ & $x_{3}$ & $x_{4}$ & $x_{5}$ & $x_{6}$ & $x_{7}$ & $x_{8}$ & $x_{9}$ & $x_{10}$ & $x_{11}$ & $x_{12}$ \\
\hline$N\left(\mu, \sum_{1}\right)$ & 0.1060 & 0 & 0.0237 & 0 & 0.2 & 0.0014 & 0.1508 & 0.0185 & 0 & 0.0215 & 0.2 & 0.0281 \\
$N\left(\mu, \sum_{2}\right)$ & 0.0570 & 0 & 0.0139 & 0 & 0.2 & 0.0155 & 0.1675 & 0.0149 & 0.0295 & 0.0224 & 0.2 & 0.0293 \\
Uniform & 0.2 & 0 & 0.0038 & 0 & 0.1266 & 0 & 0.1941 & 0.0063 & 0 & 0.0054 & 0.2 & 0.0138 \\
\hline
\end{tabular}

Table 21 Performance of CVaR model on CAR, $\beta=99 \%, \boldsymbol{J}=\mathbf{2 0 , 0 0 0}$, and MC simulation adopting $N\left(\mu, \sum_{1}\right)$

\begin{tabular}{cccccc}
\hline $\mathrm{TL}$ & Max & Mean & Min & $\leq 15 \%(\#)$ & $\leq 10.5 \%(\#)$ \\
\hline$T L_{1}(1,438,926)$ & $23.54 \%$ & $23.18 \%$ & $12.9 \%$ & 1 & 0 \\
$T L_{2}(1,463,570)$ & $21.57 \%$ & $21.2 \%$ & $10.69 \%$ & 3 & 0 \\
$T L_{3}(1,488,214)$ & $19.59 \%$ & $19.22 \%$ & $8.48 \%$ & 17 & 1 \\
$T L_{4}(1,512,858)$ & $17.58 \%$ & $17.2 \%$ & $6.27 \%$ & 21 & 3 \\
\hline
\end{tabular}


Table 22 Performance of CVaR model on CAR, $\beta=99 \%, \boldsymbol{J = 2 0 , 0 0 0}$, and MC simulation adopting $N\left(\mu, \sum_{2}\right)$

\begin{tabular}{ccccccc}
\hline TL & Max & Mean & Min & $\leq 15 \%(\#)$ & $\leq 10.5 \%(\#)$ & $\leq 8.0 \%(\#)$ \\
\hline$T L_{1}(1,438,926)$ & $23.72 \%$ & $23.39 \%$ & $16.12 \%$ & 0 & 0 & 0 \\
$T L_{2}(1,463,570)$ & $21.72 \%$ & $21.37 \%$ & $14.30 \%$ & 2 & 0 & 0 \\
$T L_{3}(1,488,214)$ & $19.71 \%$ & $19.35 \%$ & $12.20 \%$ & 15 & 0 & 0 \\
$T L_{4}(1,512,858)$ & $17.68 \%$ & $17.13 \%$ & $6.42 \%$ & 26 & 4 & 1 \\
\hline
\end{tabular}

Table 23 Performance of CVaR model on CAR, $\beta=99 \%, J=20,000$, and MC simulation adopting independent uniform distribution

\begin{tabular}{cccccc}
\hline $\mathrm{TL}$ & Max & Mean & Min & $\leq 15 \%(\#)$ & $\leq 10.5 \%(\#)$ \\
\hline$T L_{1}(1,438,926)$ & $24.03 \%$ & $23.70 \%$ & $16.95 \%$ & 0 & 0 \\
$T L_{2}(1,463,570)$ & $22.04 \%$ & $21.71 \%$ & $11.79 \%$ & 5 & 0 \\
$T L_{3}(1,488,214)$ & $20.10 \%$ & $19.71 \%$ & $8.04 \%$ & 15 & 3 \\
$T L_{4}(1,512,858)$ & $18.11 \%$ & $17.22 \%$ & $7.48 \%$ & 17 & 0 \\
\hline
\end{tabular}




\section{Appendix A: Proof of Proposition 1}

In order to keep a self-contained and convenient analysis in the context, we copy the left-hand-side item of inequality (17) with the set of probability distribution $\mathscr{D}(16)$ as follows.

$$
\min _{F \in \mathscr{D}\left(R^{n}, \mu, \rho\right)} P_{F}\left(y_{0}(x)+y(x)^{T} \xi \leq 0\right)
$$

where

$$
\mathscr{D}\left(R^{n}, \mu, \rho\right)=\left\{F \in \Xi: P\left(\xi \in R^{n}\right)=1, E_{F}\left[\xi_{k}\right]=\mu_{k}, E_{F}\left[\xi_{k}^{2}\right]=\rho_{k}, k=1,2, \ldots, n\right\}
$$

By using the probability theory, we can equivalently transform the optimization problem (17') under the constraint (16') into the following optimization problem:

$$
\begin{gathered}
\min _{F} \int_{R^{n}} \mathrm{I}_{(-\infty, 0]}\left(y_{0}(x)+y(x)^{T} \xi\right) d F(\xi) \\
\text { s.t. } \int_{R^{n}} d F(\xi)=1, \\
\quad \int_{R^{n}} \xi d F(\xi)=\mu, \\
\left\langle e_{k} e_{k}^{T}, \int_{R^{n}} \xi \xi^{T} d F(\xi)\right\rangle=\rho_{k}, k=1, \ldots, n, \\
d F(\xi) \geq 0,
\end{gathered}
$$

where $\int_{R^{n}}$ represents $n$ dimensional integral over real field. For the sake of simplicity, domain of the integral, $R^{n}$ is dropped in the remainder of this section. $\mathrm{I}_{(-\infty, 0]}$ in $(24 \mathrm{a})$ stands for the indicator function whose value

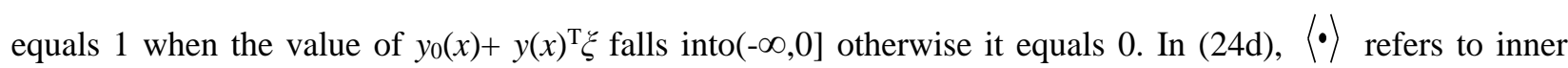
product between matrices and is equivalent to the trace of the product of two matrices; and $e_{k}$ is a $n \times 1$ unit vector where only the $k$-th entry is 1 and the remaining entries are 0 .

Define the Lagrangian function of the optimization problem (24a)-(24e) as

$$
L(\xi, \theta, \alpha, \beta)=\theta\left(1-\int d F(\xi)\right)+\alpha^{T}\left(\mu-\int \xi d F(\xi)\right)+\sum_{k=1}^{n} \beta_{k}\left(\rho_{k}-\left\langle e_{k} e_{k}^{T}, \int \xi \xi^{T} d F(\xi)\right\rangle\right)+\int 1_{(-\infty, 0]}\left(y_{0}(x)+y(x)^{T} \xi\right) d F(\xi) .
$$

Base on the above Lagrangian function, we construct the following optimization problem

$$
\min _{d F(\xi) \geq 0} \max _{\theta, \alpha, \beta} L(\xi, \theta, \alpha, \beta),
$$

where $\theta, \alpha, \beta$ are the lagrangian multipliers ( $\theta$ is a real number; and $\alpha, \beta$ are $n \times 1$ vectors).

According to Convex Analysis (Rockafellar 1970), we can easily know that the above problem (25) is equivalent to the problem (24a)-(24e). Indeed, problem (25) implies that constraints (24b)-(24d) must be satisfied. According to the dual theory from Convex Analysis (Rockafellar 1970), the dual problem for the optimization problem (25) can be written as the following form:

$\max _{\theta, \alpha, \beta} \min _{d F(\xi) \geq 0}\left\{\theta\left(1-\int d F(\xi)\right)+\alpha^{T}\left(\mu-\int \xi d F(\xi)\right)+\sum_{k=1}^{n} \beta_{k}\left(\rho_{k}-\left\langle e_{k} e_{k}^{T}, \int \xi \xi^{T} d F(\xi)\right\rangle\right)+\int 1_{(-\infty, 0]}\left(y_{0}(x)+y(x)^{T} \xi\right) d F(\xi)\right\}$

Furthermore, the optimization problem (26) can be equivalently converted as 


$$
\max _{\theta, \alpha, \beta} \theta+\alpha^{T} \mu+\beta^{T} \rho+\min _{d F(\xi) \geq 0} \int-\left(\theta+\alpha^{T} \xi+\xi^{T} \operatorname{Diag}(\beta) \xi-1_{(-\infty, 0]}\left(y_{0}(x)+y(x)^{T} \xi\right)\right) d F(\xi),
$$

where $\operatorname{Diag}(\beta)$ denotes diagonal matrix with the vector of $\beta$ being the diagonal elements. The optimization problem (27) can further be transformed to the following optimization problem:

$$
\begin{array}{cc} 
& \max _{\theta, \alpha, \beta} \quad \theta+\alpha^{T} \mu+\beta^{T} \rho \\
\text { s.t. } & \mathrm{I}_{(-\infty, 0]}\left(y_{0}(x)+y(x)^{T} \xi\right) \geq \theta+\alpha^{T} \xi+\xi^{T} \operatorname{Diag}(\beta) \xi, \quad \forall \xi \in R^{n} .
\end{array}
$$

Indeed, the constraint (29) can be equivalently transformed into the following forms:

$$
-\left(\theta+\alpha^{T} \xi+\xi^{T} \operatorname{Diag}(\beta) \xi-1_{(-\infty, 0]}\left(y_{0}(x)+y(x)^{T} \xi\right)\right) \geq 0, \quad \forall \xi \in R^{n}
$$

and

$$
-\left(\theta+\alpha^{T} \xi+\xi^{T} \operatorname{Diag}(\beta) \xi-1_{(-\infty, 0]}\left(y_{0}(x)+y(x)^{T} \xi\right)\right) d F(\xi) \geq 0,
$$

for any $d F(\xi) \geq 0$,and hence the minimum of the inner problem of the above problem (27) is zero. Therefore, the objective function of the problem (27) becomes $\theta+\alpha^{\mathrm{T}} \mu+\beta^{\mathrm{T}} \rho$ in this case.

Note that the semi-infinite constraint (29) can be easily transformed into the two equivalent semi-infinite constraints as follows:

$$
\begin{gathered}
\theta+\alpha^{T} \xi+\xi^{T} \operatorname{Diag}(\beta) \xi \leq 1, \forall \xi \in R^{n}, \\
\theta+\alpha^{T} \xi+\xi^{T} \operatorname{Diag}(\beta) \xi \leq 0 \text { for } \xi: y_{0}(x)+y(x)^{T} \xi>0 .
\end{gathered}
$$

Further, we can equivalently transform the inequality (30) into:

$$
\left[\begin{array}{cc}
\theta-1 & \frac{1}{2} \alpha^{T} \\
\frac{1}{2} \alpha & \operatorname{Diag}(\beta)
\end{array}\right] \prec 0,
$$

where “ $<0$ "stands for negative semi-definite, and equivalently transform the inequality ( 31 ) into:

$$
\max _{\xi}\left\{\theta+\alpha^{T} \xi+\xi^{T} \operatorname{Diag}(\beta) \xi: y_{0}(x)+y(x)^{T} \xi>0\right\} \leq 0 .
$$

The above constraint (32) implies that $\operatorname{Diag}(\beta)$ is a negative semi-definite matrix, hence the left-hand-side of the inequality (33) is a concave quadratic maximization problem. Furthermore, by introducing Lagrange multiplier $\lambda$ and the dual problem of the maximization problem of the left hand side of the inequality (33), the inequality (33) has the following equivalent form

$$
\min _{\lambda \geq 0} \max _{\xi}\left\{\theta+\alpha^{T} \xi+\xi^{T} \operatorname{Diag}(\beta) \xi+\lambda\left(y_{0}(x)+y(x)^{T} \xi\right)\right\} \leq 0,
$$

or

$$
\min _{\lambda \geq 0} \max _{\xi}\left\{\theta+\lambda y_{0}(x)+(\alpha+\lambda y(x))^{T} \xi+\xi^{T} \operatorname{Diag}(\beta) \xi\right\} \leq 0 .
$$

The inequality (34b) implies that there exists some $\lambda \geq 0$ and for arbitrary $\xi$ the following inequality holds

$$
\theta+\lambda y_{0}(x)+(\alpha+\lambda y(x))^{T} \xi+\xi^{T} \operatorname{Diag}(\beta) \xi \leq 0 .
$$

Furthermore, inequality (35) is equivalent to: 


$$
\left[\begin{array}{cc}
\theta+\lambda y_{0}(x) & \frac{1}{2}(\alpha+\lambda y(x))^{T} \\
\frac{1}{2}(\alpha+\lambda y(x)) & \operatorname{Diag}(\beta)
\end{array}\right] \prec 0
$$

for some $\lambda \geq 0$. In (36), “ $<0$ "stands for negative semi-definite. Through the above interpretation, the dual problem (26) or the problem (28)-(29) is equivalent to the following problem:

$$
\begin{gathered}
\max _{\theta \in R, \alpha \in R^{n}, \beta \in R^{n}, \lambda \geq 0} \theta+\alpha^{T} \mu+\beta^{T} \rho \\
\text { s.t. }\left[\begin{array}{cc}
\theta-1 & \frac{1}{2} \alpha^{T} \\
\frac{1}{2} \alpha & \operatorname{Diag}(\beta)
\end{array}\right] \prec 0 \\
{\left[\begin{array}{cc}
\theta+\lambda y_{0}(x) & \frac{1}{2}(\alpha+\lambda y(x))^{T} \\
\frac{1}{2}(\alpha+\lambda y(x)) & \operatorname{Diag}(\beta)
\end{array}\right] \prec 0 .}
\end{gathered}
$$

The remaining job in order to complete the proof of Proposition 1, is to prove that the primal problem (24a)-(24e) or (25) and its dual problem (26) is equivalent. We set $\varphi=\mathrm{I}_{(-\infty, 0]}\left(y_{0}(x)+y(x)^{\mathrm{T}} \xi\right), \psi_{1}(\cdot)=1, b_{1}=1, \psi_{2}(\cdot)=$ $\xi, b_{2}=\mu, \psi_{i}(\cdot)=\xi_{i-2}{ }^{2}, b_{i}=\rho_{i-2}(i=3, \ldots, n+2)$. We can easily prove that functions $\psi_{2}, \ldots, \psi_{n+2}$ are continuous and $\varphi$ is upper semicontinuous. Further, we can know that the primal problem is consistent. According to the Corollary 3.1 of Shapiro (2001), we can prove that there is no gaps between the primal optimization problem (24a)-(24e) and it's dual optimization problem (26). Based on the above analysis, we can prove that the chance constraint (17) with the uncertain set (16) is equivalent to

$$
\max _{\theta, \alpha, \beta, \lambda \geq 0}\left\{\theta+\alpha^{T} \mu+\beta^{T} \rho:\left[\begin{array}{cc}
\theta-1 & \frac{1}{2} \alpha^{T} \\
\frac{1}{2} \alpha & \operatorname{Diag}(\beta)
\end{array}\right] \prec 0,\left[\begin{array}{cc}
\theta+\lambda y_{0}(x) & \frac{1}{2}(\alpha+\lambda y(x))^{T} \\
\frac{1}{2}(\alpha+\lambda y(x)) & \operatorname{Diag}(\beta)
\end{array}\right] \prec 0\right\} \geq 1-\varepsilon .
$$

Further, by using a simple analysis, the inequality (38) can be equivalently rewritten as follows: there exist $\theta \in$ $R, \alpha \in R^{n}, \beta \in R^{n}$ and $\lambda \geq 0$ such that $\theta+\alpha^{\mathrm{T}} \mu+\beta^{\mathrm{T}} \rho \geq 1-\varepsilon$ and constraints (37b)- (37c) are satisfied. Thus, the proof of Proposition 1 is completed.

\section{Appendix B: The Proof of Theorem 1}

Let $\theta^{*}=\theta / \lambda, \alpha^{*}=\alpha / \lambda, \beta^{*}=\beta / \lambda$ and $\lambda^{*}=1 / \lambda,(19 \mathrm{a})-(19 \mathrm{c})$ in proposition 1 are equivalently transformed to (21b)-(21d) in theorem 1 for the case of $\lambda>0$ and $x, \theta^{*}, \alpha^{*}, \beta^{*}$ and $\lambda^{*}(>0)$ become decision variables. In fact, the constraint (34a) or (34b) implies that there exists some $\lambda>0$ such that

$$
\max _{\xi}\left\{\theta+\lambda y_{0}(x)+(\alpha+\lambda y(x))^{T} \xi+\xi^{T} \operatorname{Diag}(\beta) \xi\right\} \leq 0,
$$

otherwise for any $\lambda>0$,

$$
\max _{\xi}\left\{\theta+\lambda y_{0}(x)+(\alpha+\lambda y(x))^{T} \xi+\xi^{T} \operatorname{Diag}(\beta) \xi\right\}>0 .
$$

If (39) holds for any $\lambda>0$, then the minimization of the problem described as the left-hand-side of inequality (34a) can be obtained only when $\lambda=0$; thus inequality (34) is equivalent to 


$$
\max _{\xi}\left\{\theta+\alpha^{T} \xi+\xi^{T} \operatorname{Diag}(\beta) \xi\right\} \leq 0
$$

i.e.,

$$
\left[\begin{array}{cc}
\theta & \frac{1}{2} \alpha^{T} \\
\frac{1}{2} \alpha & \operatorname{Diag}(\beta)
\end{array}\right] \prec 0,
$$

and the equivalent form (38) of CAR constraint (17) or (15) becomes

$$
\max _{\theta, \alpha, \beta}\left\{\theta+\alpha^{T} \mu+\beta^{T} \rho:\left[\begin{array}{cc}
\theta & \frac{1}{2} \alpha^{T} \\
\frac{1}{2} \alpha & \operatorname{Diag}(\beta)
\end{array}\right] \prec 0\right\} \geq 1-\mathcal{E},
$$

which implies CAR constraint has relation with lagrangian multipliers but no any relation with investment proportions $x$ i.e., there is virtually no restriction on the optimal investment proportions of CAR constraint. Therefore, the constraint (19c) in Proposition 1 for some $\lambda>0$ is required from practical perspective and it is equivalent to the constraint (21d). Besides, the equivalence of (19a) and (21b), (19b) and (21c) is obvious, if let $\theta^{*}=\theta / \lambda, \alpha^{*}=\alpha / \lambda, \beta^{*}=\beta / \lambda$ and $\lambda^{*}=1 / \lambda$. Finally, combining the objective function (14), the transformed CAR constraints (21b)-(21d) and other constraints (20), the asset allocation optimization model is reformulated as tractably linear SDP problem as (21a)-(21g). The proof of Theorem 1 is completed. 